\title{
Genome-wide maps of enhancer regulation connect risk variants to disease genes
}

Joseph Nasser ${ }^{1, *}$, Drew T. Bergman ${ }^{1, *}$, Charles P. Fulco ${ }^{1,24, *}$, Philine Guckelberger ${ }^{1,2, *}$, Benjamin R. Doughty ${ }^{1, *}$, Tejal A. Patwardhan ${ }^{1,3}$, Thouis R. Jones ${ }^{1}$, Tung H. Nguyen ${ }^{1}$, Jacob C. Ulirsch ${ }^{1,4}$, Heini M. Natri, ${ }^{5,6}$, Elle M. Weeks ${ }^{1}$, Glen Munson ${ }^{1}$, Michael Kane ${ }^{1}$, Helen Y. Kang ${ }^{7,8}$, Ang Cui1,9, John P. Ray ${ }^{1}$, Tom M. Eisenhaure ${ }^{1}$, Kristy Mualim ${ }^{7}$, Ryan L. Collins ${ }^{1,10,11}$, Kushal Dey ${ }^{12}$, Alkes L. Price $1,12,13$, Charles B. Epstein ${ }^{1}$, Anshul Kundaje ${ }^{7,14}$, Ramnik J. Xavier $^{1,15-17}$, Mark J. Daly ${ }^{1,18-20}$, Hailiang Huang ${ }^{1,18,19}$, Hilary K. Finucane ${ }^{1,18,19}$, Nir Hacohen ${ }^{1,19,21}$, Eric S. Lander $^{1,22,23, \dagger}$, Jesse M. Engreitz ${ }^{1,7,8 \dagger}$

${ }^{1}$ Broad Institute of MIT and Harvard, Cambridge, MA

2Department of Biology, Chemistry, and Pharmacy, Freie Universität Berlin, Berlin, Germany

${ }^{3}$ Department of Statistics, Harvard University, Cambridge, MA

${ }^{4}$ Program in Biological and Biomedical Sciences, Harvard Medical School, Boston, MA, USA

${ }^{5}$ Center for Evolution and Medicine, School of Life Sciences, Arizona State University, Tempe, AZ

${ }^{6}$ The Translational Genomics Research Institute, Phoenix, AZ

${ }^{7}$ Department of Genetics, Stanford University School of Medicine, Stanford, CA

${ }^{8}$ BASE Initiative, Betty Irene Moore Children's Heart Center, Lucile Packard Children's Hospital, Stanford University School of

Medicine, Stanford, CA

${ }^{9}$ Harvard-MIT Division of Health Sciences and Technology, MIT, Cambridge, MA

${ }^{10}$ Program in Bioinformatics and Integrative Genomics, Harvard Medical School, Boston, MA

${ }^{11}$ Center for Genomic Medicine, Massachusetts General Hospital, Boston, MA

${ }^{12}$ Department of Epidemiology, Harvard T. H. Chan School of Public Health, Boston, MA, USA

${ }^{13}$ Department of Biostatistics, Harvard T.H. Chan School of Public Health, Boston, MA

${ }^{14}$ Department of Computer Science, Stanford University, Stanford, CA

${ }^{15}$ Center for Computational and Integrative Biology, Massachusetts General Hospital, Harvard Medical School, Boston, MA

${ }^{16}$ Department of Molecular Biology, Massachusetts General Hospital, Harvard Medical School, Boston, MA

${ }^{17}$ Gastrointestinal Unit and Center for the Study of Inflammatory Bowel Disease, Massachusetts General Hospital, Boston, MA

${ }^{18}$ Analytic and Translational Genetics Unit, Massachusetts General Hospital, Boston, MA

${ }^{19}$ Department of Medicine, Harvard Medical School, Boston, MA

${ }^{20}$ Institute for Molecular Medicine Finland, University of Helsinki, Helsinki, Finland

${ }^{21}$ Center for Cancer Research, Massachusetts General Hospital, Boston, MA

${ }^{22}$ Department of Biology, MIT, Cambridge, MA

${ }^{23}$ Department of Systems Biology, Harvard Medical School, Boston, MA

${ }^{24}$ Present address: Bristol Myers Squibb, Cambridge, MA

*These authors contributed equally as first authors

tThese authors jointly supervised the work

Abstract: Genome-wide association studies have now identified tens of thousands of noncoding loci associated with human diseases and complex traits, each of which could reveal insights into biological mechanisms of disease. Many of the underlying causal variants are thought to affect enhancers, but we have lacked genome-wide maps of enhancer-gene regulation to interpret such variants. We previously developed the Activity-by-Contact (ABC) Model to predict enhancer-gene connections and demonstrated that it can accurately predict the results of CRISPR perturbations across several cell types. Here, we apply this ABC Model to create enhancer-gene maps in 131 cell types and tissues, and use these maps to interpret the functions of fine-mapped GWAS variants. For inflammatory bowel disease (IBD), causal variants are $>20$-fold enriched in enhancers in particular cell types, and $A B C$ outperforms other regulatory methods at connecting noncoding variants to target genes. Across 72 diseases and complex traits, ABC links 5,036 GWAS signals to 2,249 unique genes, including a class of 577 genes that appear to influence multiple phenotypes via variants in enhancers that act in different cell types. Guided by these variant-to-function maps, we show that an enhancer containing an IBD risk variant regulates the expression of PPIF to tune mitochondrial membrane potential. Together, our study reveals insights into principles of genome regulation, illuminates mechanisms that influence IBD, and demonstrates a generalizable strategy to connect common disease risk variants to their molecular and cellular functions. 


\section{Introduction}

Genome-wide association studies (GWAS) have now identified tens of thousands of genetic variants that influence risk for human diseases and traits ${ }^{1}$. Each of these associations could provide insight into biological mechanisms underlying human disease by pinpointing a particular variant, gene, and cell type that influences disease. For example, studies of protein-coding variants associated with inflammatory bowel diseases (IBD) have uncovered causal roles for IL23R via pro-inflammatory signaling in $\mathrm{T}^{\text {cells }}{ }^{2}$, C1ORF106 in barrier function in enterocytes ${ }^{3}$, and ATG16L1 in autophagy in a variety of immune and epithelial cells ${ }^{4-7}$ - thereby identifying new pathways and therapeutic targets ${ }^{8}$. Yet, beyond these and a handful of other examples ${ }^{9-12}$, connecting GWAS associations to molecular functions has proven challenging.

Three obstacles have blocked progress. One challenge is that each GWAS signal might include dozens of variants in linkage disequilibrium with one another that tag a single causal variant. The second challenge is that most causal variants for common diseases do not directly alter proteincoding sequences and instead occur in noncoding gene regulatory elements such as enhancers, which can regulate genes over long genomic distances ${ }^{13,1417,18}$. Finally, many enhancers appear to act in very specific cell types or cell states ${ }^{15,16}$, making it challenging to identify the particular cellular context in which a disease variant might act. As such, studying any given GWAS association remains daunting because it can include many possible variants, dozens of possible target genes, and dozens of possible cell types and states relevant to disease ${ }^{1,19}$.

Recent developments set the stage for addressing these challenges. First, toward distinguishing among multiple possible variants in a locus, recent studies have applied genetic fine-mapping methods to prioritize likely causal variants for many GWAS signals ${ }^{20-23}$. For example, finemapping identified 45 variants for IBD with at least $50 \%$ probability of causing an association signal, and 364 variants with at least $10 \%$ probability $^{21}$. Second, we recently developed a computational approach called the Activity-by-Contact $(A B C)$ Model to identify enhancers in a particular cell type and predict their target genes based on maps of chromatin state and 3D folding ${ }^{24}$. This method could allow us to build genome-wide maps of enhancer-gene regulation that describe which enhancers regulate which genes in which cell types. Together, these advances suggest a new opportunity to connect noncoding GWAS variants to their target genes and cell types.

Here, we build $A B C$ enhancer-gene maps in 131 biosamples spanning 74 distinct cell types and tissues, and use these maps to analyze fine-mapped genetic variants associated with 72 diseases and complex traits. We find that $40 \%$ of likely causal noncoding GWAS variants (fine-mapping posterior probability $>95 \%$ ) overlap $A B C$ enhancers in these cell types. For IBD, ABC links noncoding variants to 43 genes in an array of immune cell types, identifying context-specific functions for new and known genes and pathways. Together, our study provides a map of enhancer-gene regulation across cell types, demonstrates a generalizable strategy to connect thousands of risk variants to target genes, and provides a foundation for studying the molecular mechanisms that influence IBD and other common diseases.

\section{Results}

\section{Genome-wide maps of enhancers and target genes in 131 biosamples}

We used the ABC Model $^{24}$ to construct genome-wide maps of enhancer-gene connections across 131 biosamples. These maps combine data from the ENCODE Consortium and other sources (in primary cell types, tissues, and immortalized or transformed cell lines) with new datasets we collected (in immune cell lines amenable to CRISPR experiments) (Table S1, Table S2, Fig. 1a). 
For each biosample, we defined a set of candidate elements by calling peaks in chromatin accessibility data (DNase-seq or ATAC-seq). For each candidate element and nearby active promoter, we calculated an ABC score by multiplying enhancer activity (defined in terms of chromatin accessibility and H3K27ac ChIP-seq signals in a given biosample) and elementpromoter 3D contact frequency (estimated using a common $\mathrm{Hi}-\mathrm{C}$ dataset averaged across 10 human biosamples) (see Methods) ${ }^{24}$. We defined candidate element-gene pairs that exceeded a chosen threshold on the ABC score as "enhancer-gene connections", and elements predicted to regulate at least one gene as "ABC enhancers" (see Methods).

Across 131 biosamples, we identified 6,316,021 enhancer-gene connections for 23,219 expressed genes and 269,539 unique enhancers. In a given biosample, ABC identified an average of 48,441 enhancer-gene connections for 17,605 unique enhancers, comprising $\sim 2.9 \mathrm{Mb}$ of enhancer sequence ( $12 \%$ of chromatin-accessible regions, $0.11 \%$ of the mappable genome, Table S2). Each enhancer regulated an average of 2.7 genes, and each gene was regulated by 2.8 enhancers (Fig. S1). Enhancer-gene connections were very different between biosamples (on average, only $19 \%$ of enhancer-gene connections are shared between pairs of biosamples, versus $85 \%$ for biological replicates of the same biosample, see Fig. S2).

We compared these $A B C$ predictions to an expanded compendium of CRISPR perturbation data including 5,755 tested distal element-gene pairs in 11 cell types and states (Tables S3, S4). ABC performed well at classifying regulatory connections (area under the precision-recall curve $(A U P R C)=0.64)$, and outperformed other methods based on assigning elements to the closest gene (AUPRC $=0.16$ ), analyzing 3D loops or domains (best AUPRC $=0.05$ ), correlating enhancer activity with promoter activity or gene expression (best AUPRC $=0.15$ ), or other computational prediction methods (best AUPRC $=0.22$ ), similar to our previous observations using a subset of this CRISPR data ${ }^{24}$ (Fig. S3, Table S5, see Methods).

\section{GWAS variants are strongly enriched in $A B C$ enhancers}

Noncoding GWAS variants are thought to regulate gene expression by affecting regulatory elements in particular cell types ${ }^{13,14}$, yet we have lacked accurate maps to quantify the enrichment of causal variants in enhancers. We examined how frequently genome-wide significant GWAS variants for IBD and 71 other traits overlap ABC enhancers in each biosample (Table S6). To overcome the challenge of identifying causal variants at each GWAS locus, we leveraged finemapping analyses that assigned to each variant a posterior inclusion probability (PIP) of causality and defined $95 \%$ credible sets for conditionally independent signals (here, defined as the minimal set of variants whose PIP sum to at least $95 \%$, see Methods) ${ }^{21}$. To focus on higher-confidence distal regulatory signals, we examined the 24,922 fine-mapped variants with PIP $\geq 10 \%$ in credible sets that did not contain any coding or splice site variant $(37-100 \%$ of credible sets for these traits, Fig. S4a).

The fine-mapped GWAS variants showed striking enrichments (up to 48-fold) in ABC enhancers in certain cell types, with the strongest enrichments typically occurring in cell types with biological relevance to the trait, and showed much stronger enrichments in $A B C$ enhancers than in other chromatin accessible regions (Fig. 1b,c). For example, fine-mapped variants for 4 blood cell traits (monocyte, lymphocyte, erythrocyte, and platelet counts) showed strong enrichments in ABC enhancers in the corresponding cell type (11- to 16-fold compared to all variants in 1000 Genomes Version 3), and were more strongly enriched in the corresponding cell type than in other blood or unrelated cell types (Fig. S4b). In total, fine-mapped variants for 65 of 72 traits were significantly enriched in $\mathrm{ABC}$ enhancers in at least one biosample (Table S7). 
For IBD, fine-mapped variants were significantly enriched in $A B C$ enhancers in 65 biosamples (Bonferroni-corrected Fisher's exact test $P<0.001$ ), including 56 immune cell types/cell lines and tissue from the large intestine (Fig. 1b; Table S6). The top biosample showed 21-fold enrichment and corresponded to activated dendritic cells, which are known to play an important role in the initiation of inflammation in IBD ${ }^{8,25,26}$ (19 of 285 fine-mapped IBD variants (6.7\%) overlapped ABC enhancers in dendritic cells, compared to $0.3 \%$ of all common variants; this included variants in 15 of 93 credible sets). Across all biosamples, fine-mapped variants were on average 2.3-fold more strongly enriched in $A B C$ enhancers than in other chromatin accessible regions not called as enhancers by the ABC Model (two-sided signed-rank test $P<10^{-15}$, Fig. 1b).

We observed even stronger enrichments when we used stratified linkage disequilibrium score regression (S-LDSC) to estimate the heritability enrichment in ABC enhancers (Table S6). (SLDSC considers not only variants in genome-wide significant GWAS loci but also in subsignificant loci). For example, S-LDSC found 50 -fold enrichment for erythrocyte count heritability in $A B C$ enhancers in erythroblasts and 42-fold enrichment for IBD heritability in ABC enhancers in dendritic cells.

\section{Enhancers likely contain a majority of causal noncoding GWAS variants}

We next examined the total fraction of fine-mapped noncoding variants that overlapped $A B C$ enhancers, as opposed to other noncoding sequences such as splice sites. Across all signals for these 72 traits, $A B C$ enhancers contained $40 \%$ of the 2,520 noncoding variants with PIP $>=95 \%$, compared to $7.5 \%$ of all common noncoding variants (Fig. 1c, Fig. S4c). For 12 blood cell traits, where the relevant cell types are better represented in our dataset, $A B C$ enhancers contained $46 \%$ of 722 noncoding variants with PIP $>=95 \%$ (Fig. 1C). For IBD, ABC enhancers contained 4 of 10 noncoding variants with PIP $\geq 95 \%$ and 98 of 344 noncoding variants with PIP $>=10 \%$, including variants in 54 of 93 noncoding IBD credible sets (57\%).

Importantly, our analysis underestimates the proportion of fine-mapped variants residing in $A B C$ enhancers because we still lack appropriate data for many relevant cell types. For IBD, for example, the maps are missing at least half of the $>50$ distinct cell types in the intestinal lamina propria $^{27}$, including cell types known to be important for IBD (e.g., Goblet and Paneth cells ${ }^{8}$ ). We anticipate that, when $A B C$ maps are expanded to include hundreds of additional cell types, a majority of causal noncoding GWAS variants will reside in $A B C$ enhancers (Fig. S4d). 
a

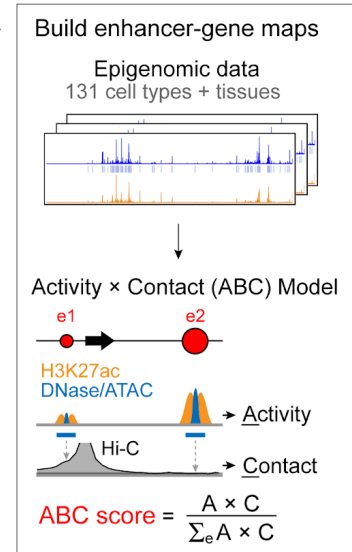

b
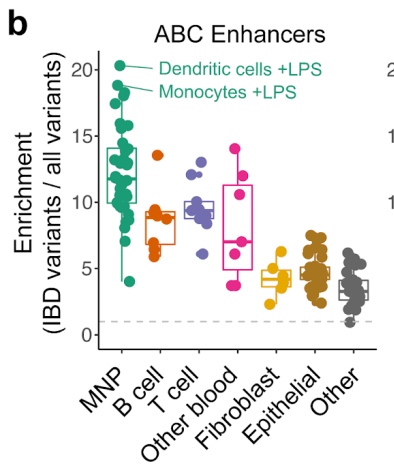

d

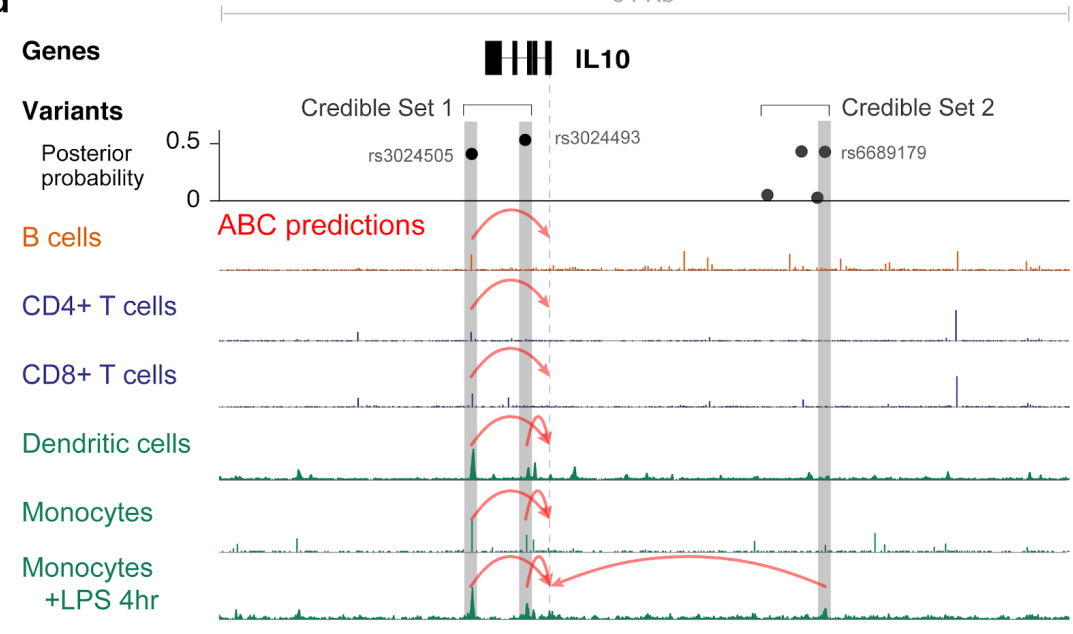

Figure 1. Enhancer-gene maps connect finemapped variants to enhancers, genes, and cell types.

(a) Overview of approach.

(b) Enrichment of fine-mapped IBD variants (PIP $>=10 \%$ ) in $A B C$ enhancers (left) and all other accessible regions (right) in each of 131 biosamples. $A B C$ enhancers in mononuclear phagocytes (MNPs) including dendritic cells and monocytes stimulated with LPS show the strongest enrichments, and $A B C$ enhancers (the subset of accessible regions predicted by $A B C$ to regulate at least one gene) show much stronger enrichments than the other accessible regions not called as ABC enhancers.

(c) Fraction of noncoding variants above a given PIP threshold that overlap an $A B C$ enhancer in any biosample. Black line shows the weighted average across 72 traits. Traces are shown for PIP thresholds above which there are at least 5 variants. Dashed line shows the fraction of all common noncoding variants that overlap $A B C$ enhancers.

(d) $\mathrm{ABC}$ predictions connect two IBD GWAS signals to IL10. Signal tracks show DNase- or ATAC-seq (based on availability of data). Red arrows represent $A B C$ predictions connect variants to IL10. Dashed line shows transcription start site (TSS). Gray bars highlight fine-mapped variants that overlap ABC enhancers in at least one cell type. Credible set 1 contains two variants, both of which overlap enhancers predicted to regulate $I L 10$ in various cell types. Credible set 2 contains four variants, one of which overlaps an enhancer predicted to regulate IL10 in monocytes stimulated with LPS. 


\section{ABC maps connect GWAS variants to disease genes}

Variants in enhancers influence disease through effects on the expression of nearby genes. Detailed experimental studies have demonstrated that individual noncoding variants can affect the regulation of one or more genes over long distances ${ }^{10,11,28}$. Yet, it has proven challenging to accurately measure or predict which gene(s) are regulated by a given variant, and which effects are relevant to disease $e^{29-33}$.

We tested how well ABC enhancer-gene maps could connect noncoding GWAS signals to target genes for IBD (Fig. 1a, Note S1). For each credible set for a trait, we intersected fine-mapped variants ( $P I P \geq 10 \%$ ) with $A B C$ enhancers in biosamples globally enriched for such overlaps, and assigned the credible set to the target gene(s) predicted by $A B C$. In cases where multiple variants overlapped enhancers or an enhancer regulated multiple genes, we assigned the credible set to the single gene with the highest $A B C$ score ("ABC-Max"). For example, Fig. 1d depicts two independent IBD credible sets in the $1 q 32.1$ locus. Both credible sets include noncoding variants with PIP $\geq 10 \%$ that overlap $\mathrm{ABC}$ enhancers in monocytes stimulated with bacterial lipopolysaccharide (LPS), the biosample with the second highest enrichment for IBD (Fig. 1c). For both credible sets, ABC-Max predicted that these enhancers regulate multiple genes in the locus, but the gene with the highest $A B C$ score was IL10, a key anti-inflammatory cytokine known to be important for IBD ${ }^{8}$ (Fig. 1d, Fig. S5a). Notably, ABC identified IL10 but not two neighboring genes in the same interleukin family, IL19 and IL20 (Fig. S5a).

To systematically evaluate the predictions of ABC-Max and benchmark it against other approaches, we first examined a curated set of 64 known IBD genes previously identified based on associated coding variants or evidence regarding the effect of the gene in experimental models $^{8}$ (see Methods, Table S8). We analyzed the 37 noncoding credible sets within $1 \mathrm{Mb}$ of exactly one of these genes, and tested how often ABC-Max or other methods identified the known gene and distinguished it from the other genes within $1 \mathrm{Mb}$ (median: 13 genes; range: 3-66). We visualized performance using a precision-recall plot, where recall is the fraction of credible sets for which the known gene is identified (sensitivity), and precision is the fraction of predicted genes corresponding to known genes (positive predictive value) (Fig. 2a).

As a baseline, we tested the simple heuristic of assigning each GWAS credible set to the closest gene. This nearest-gene method does not define a regulatory link from variant to enhancer to gene and cell type, but has been shown to assign $\sim 70 \%$ of metabolite GWAS loci to genes with plausible biochemical functions ${ }^{34}$. In our IBD dataset, connecting the lead variant for each of the 37 IBD credible sets to the closest gene transcription start site (TSS) correctly identified the known IBD gene in 27 of 37 cases (73\% precision, Fig. $2 \mathbf{a})$.

We next evaluated previous methods or predictions to connect regulatory variants to disease genes, including eQTL colocalization ${ }^{35,36}$, transcriptome-wide association studies ${ }^{32}$, Mendelian Randomization ${ }^{37}$, promoter-capture $\mathrm{Hi}-\mathrm{C}^{27}$, and alternative enhancer-gene prediction methods ${ }^{16,38-}$ ${ }^{44}$ (see Methods). For nearly all methods, choosing the gene with the best score in each locus, rather than choosing all genes above the global threshold previously reported by these studies, obtained much higher precision (Fig. S5b). Still, most of these methods performed poorly (Fig. 2a). One eQTL analysis ${ }^{35}$ achieved $100 \%$ precision, but made predictions in only 2 loci (2 of 2 correct). The enhancer-gene prediction method with the highest precision (linking enhancers to target genes based on correlating chromatin state with gene expression across cell types from the Roadmap Epigenomics Project ${ }^{38,45}$ ) made 22 predictions and identified the known IBD genes in 16 of 22 cases ( $72 \%$ precision). (However, this correlation method performed much less well at predicting the results of CRISPR perturbations (AUPRC $<0.15$, Fig. S3)). 
Finally, we evaluated the predictions of ABC-Max. Of the 37 credible sets, 18 included a variant that overlapped an $\mathrm{ABC}$ enhancer in an enriched biosample, and ABC-Max identified the known gene in 17 of 18 cases (94\% precision) (Fig. 2a). Thus, ABC-Max identifies a high-confidence set of genes at these IBD GWAS loci, with higher precision than other enhancer prediction methods. The fraction of loci with a prediction (recall $=49 \%$ ) would likely increase upon expanding the $A B C$ maps to include additional relevant cell types in the gut.

We performed a second analysis to compare ABC-Max to other methods for identifying genes for IBD. We analyzed all 93 fine-mapped noncoding credible sets (regardless of whether they contained a known IBD gene) and examined the extent to which the genes nominated by each method were enriched in gene sets from the Molecular Signatures Database (see Methods) ${ }^{46}$. Genes identified by ABC-Max for IBD showed stronger gene-set enrichments than the genes identified by other regulatory methods or by closest gene (Fig. 2b).

Toward the goal of understanding the good performance of ABC-Max, we made two observations. First, assigning each credible set to the gene with the strongest ABC score ("ABC-Max"; precision $=94 \%$ for known IBD genes) performed far better than assigning each credible set to all genes linked to an IBD variant ("ABC-All"; precision =17\%) (Fig. S5b). This was because individual noncoding variants often overlapped $A B C$ enhancers that were predicted to regulate multiple genes (median: 3, range: 1-17), with the known gene having the highest ABC score (e.g., Fig. S5a). This complexity appears to be a fundamental feature of mammalian gene regulation: ciseQTL studies indicate that noncoding variants often regulate multiple genes in a given cell type ${ }^{47}$, and CRISPR experiments have identified individual enhancers that regulate up to 8 genes in $\operatorname{cis}^{24,48}$. Our observations are consistent with the idea that, while variants often affect the expression of multiple genes, only a subset of these effects are likely relevant to disease ${ }^{31}$.

Second, the recall of ABC-Max depended on the inclusion of relevant cell types in the analysis. To illustrate this, we repeated our $A B C$ predictions after removing all immune cell types and gut tissue samples from our compendium. With this more limited set of samples (65 of 131 total), the recall of ABC-Max dropped from $46 \%$ to $24 \%$ (Fig. S5b). (The 9 genes correctly identified in this reduced dataset all appeared to be explained by variants in enhancers that were active in multiple cell types - for example, in both gut tissue and in adrenal gland, another epithelial tissue.) This highlights one feature of $A B C$, relative to $\mathrm{eQTL}$ approaches: due to the modest data requirements of ABC (chromatin accessibility and H3K27ac ChIP-seq data from a single individual), it is possible to explore a broad array of cell types and states relevant to disease.

Together, these analyses demonstrate that $A B C$ maps can accurately connect fine-mapped variants to target genes for a substantial fraction of IBD GWAS signals, and thereby provide hypotheses for regulatory mechanisms linking causal variants to enhancers to cell types to genes. 

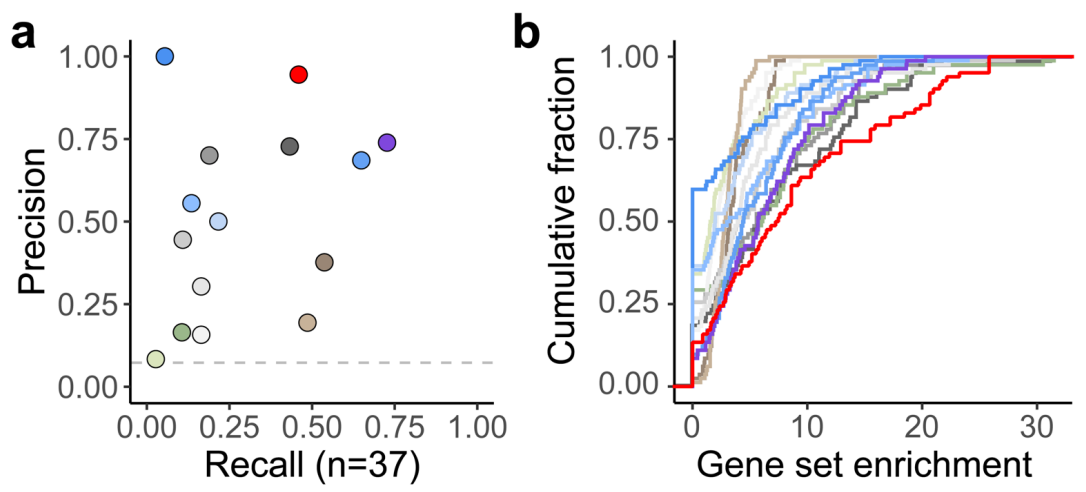

Method:
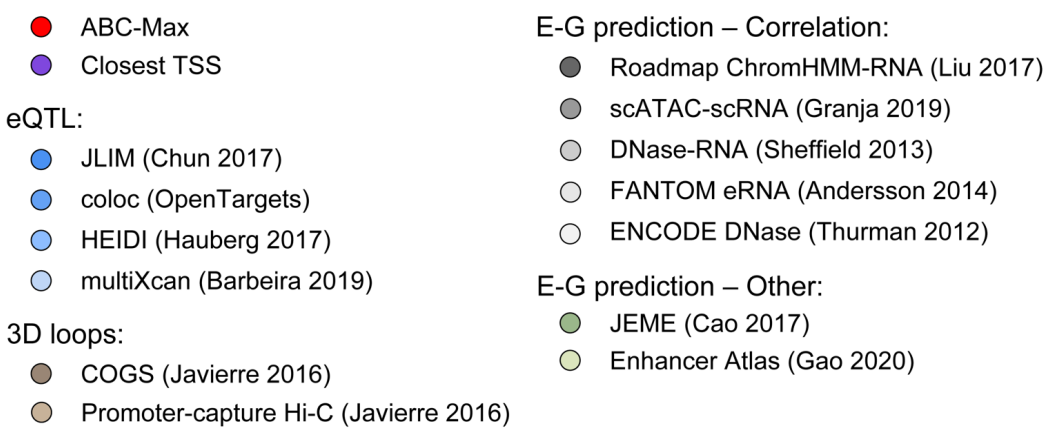

Fig. 2. $A B C$ enhancer maps identify known genes for IBD.

(a) Precision versus recall for methods to connect noncoding IBD credible sets to known IBD disease genes ${ }^{8}$, considering 37 credible sets with exactly 1 known gene within $1 \mathrm{Mb}$. Precision $=$ fraction of identified genes corresponding to known genes. Recall = fraction of the 37 known genes identified. See Methods for details on approaches. Where quantitative scores were available (e.g., colocalization probability for eQTL coloc), plot presents the performance of choosing the gene with the best score per locus, which outperforms selecting all genes above a global threshold (see Fig. S5b).

(b) Cumulative density plot showing enrichment for gene sets in MSigDB among the genes prioritized by each method ${ }^{46}$. For each method, we first identified the top 5 most enriched significant gene sets in the predictions of that method ( 82 gene sets total). Then, we calculated the levels of enrichment of all 82 gene sets in the predictions of each method. 


\section{Context-specific functions for IBD genes and pathways}

We next explored how applying $A B C$ maps to many GWAS loci for a disease might identify genes and pathways that act in specific cellular contexts. To do so, we examined the 47 predictions made by ABC-Max for noncoding IBD credible sets, which nominated 43 unique IBD genes (Fig. 3a, Table S9). The distance from the noncoding variant in the ABC enhancer to the TSS of the predicted target gene ranged from $73 \mathrm{bp}$ to $804 \mathrm{~Kb}$ (median: $18 \mathrm{~Kb}$ ), and 11 of 47 predictions involved a gene that was not the closest (Fig. 3a,b).

ABC-Max identified both known and novel IBD genes. Among the 43 prioritized IBD genes, many have previously reported functions in immunity and inflammation, and were enriched for genes in the interferon gamma pathway (6 genes; 12-fold enrichment), lymphocyte activation (11 genes; 7-fold enrichment), and regulation of transcription from RNA polymerase II promoter (21 genes; 5 -fold enrichment), among other gene sets (Fig. 3a). For at least 26 of these genes, knockouts or other genetic models have been shown to affect the severity of experimental colitis in mice (Table S10). Yet, only 15 of the prioritized IBD genes were previously reported as implicated in IBD risk in humans ${ }^{8,49}$, and only 20 have been previously linked to IBD risk variants in large eQTL or TWAS studies (Fig. 3a, Table S8) $32,35-37,50$. The 14 newly prioritized genes included PPIF, a gene involved in mitochondrial metabolism whose function in immune cells is not well understood (see below).

The cell-type specificity of ABC enhancer-gene connections allowed us to categorize GWAS loci by predicted cell type(s), often identifying a cellular context more specific than the expression pattern of the gene itself (Fig. 3a,c, Fig. S6). For example, IL6ST is expressed in most cell types, but the linked IBD risk variant (rs7731626, PIP $=28 \%$ ) overlapped an enhancer that was active only in T cell subsets and fetal thymus tissue (Fig. S6d). To validate this prediction, we used CRISPRi-FlowFISH ${ }^{24}$ to test whether the predicted enhancer regulates IL6ST expression in four immune cell lines representative of T cell, B cell, and monocytic lineages. Although IL6ST was expressed in each cell line, the enhancer regulated IL6ST expression only in a T cell line (Jurkat), and not in B cell or monocytic cell lines (GM12878, BJAB, and THP1) (Fig. S6d). Together, these data suggest that this IBD risk variant affects gene expression in $\mathrm{T}$ cells, and not in other cell types. More generally, for the $47 \mathrm{ABC}-$ Max predictions, the promoter of the target gene was active in a median of 6 of 6 biosample/cell type categories (e.g., T cells, B cells, see Fig. 3a), but the enhancer was predicted to regulate that gene in a median of only 2 categories (Fig. $3 \mathbf{c}$, two-sided rank-sum test $P<10^{-16}$ ).

Connecting GWAS loci to effects in particular cell types could improve the identification of gene pathways relevant to disease. For example, the 23 IBD genes predicted in mononuclear phagocytes (MNPs: monocytes, macrophages, and dendritic cells) included a cascade of known cell-surface receptors and co-stimulatory molecules (CD40, HLA-DQA1, IFNGR2, IL2RA, CCR6), signal transduction regulators (KSR1, SOCS1), transcriptional regulators (including IKZF1, ERG, IRF4, RORC), and downstream secreted immunomodulators (PDGFB, CCL2, IL10). Overall, the 23 IBD genes predicted by $A B C$ in MNPs showed much stronger gene-set enrichments in the Molecular Signatures Database (see Methods) than did the 43 IBD genes predicted without regard to cell type (up to 27- vs 16-fold enrichment, respectively; two-sided rank-sum $P<0.002$, Fig. 3d), supporting the idea that grouping genes by relevant cell type can facilitate the identification of gene networks relevant to disease ${ }^{27,51,52}$. 
a
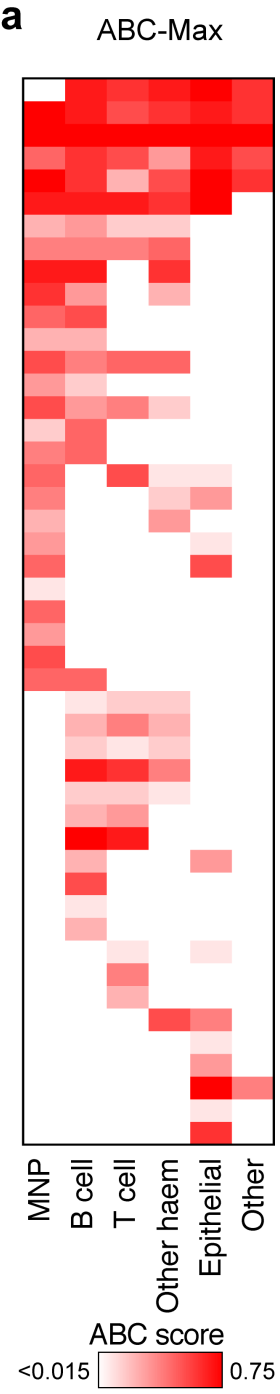

Distar

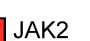
IFNGR2
FUBP1*
BCL9L**
SOCS1 SOCS1

SMAD7* PMIF* NCF4 CD40 IKZF1 IL2RA IL2RA
IL2RA
IL 10
IL 10 IL10 IL10 RORC RORC SBNO2 SMAD3 KSR1 HLA-DQA1 CCL2 ERG*
PDGFB
PDGFB PDGFB CEBPB
TAF8*
ADO ADO C1orf106 PTPN2 IRF8 TNFSF15 EGR2* GPR18 MYC IL6ST JAZF1 NKX2-3 RASL11A RNF186 RNF186 SLC26A3 istance Known eQTL/ Enriched Genes TWAS Gene Sets

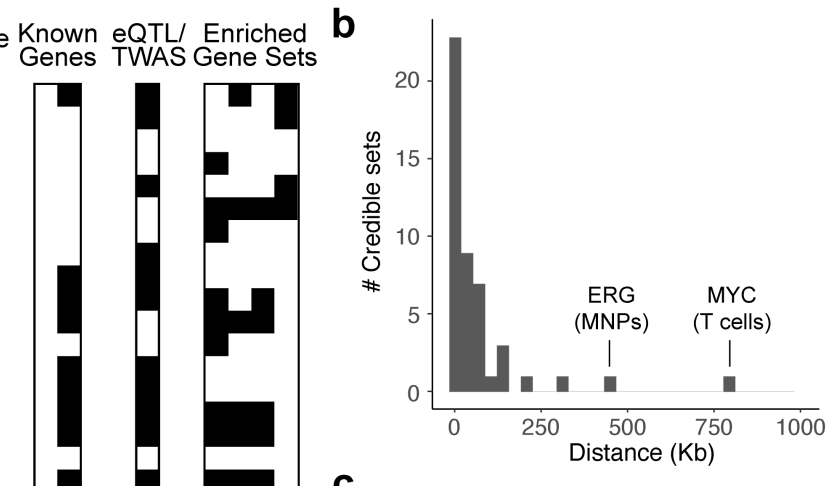

C

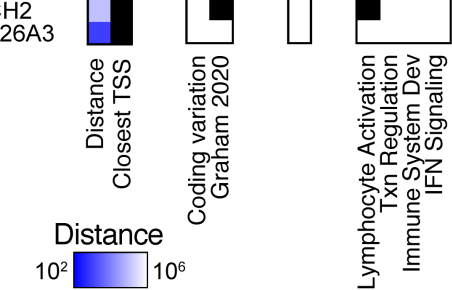

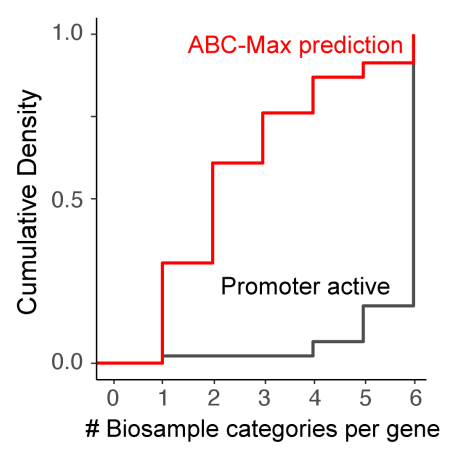

d

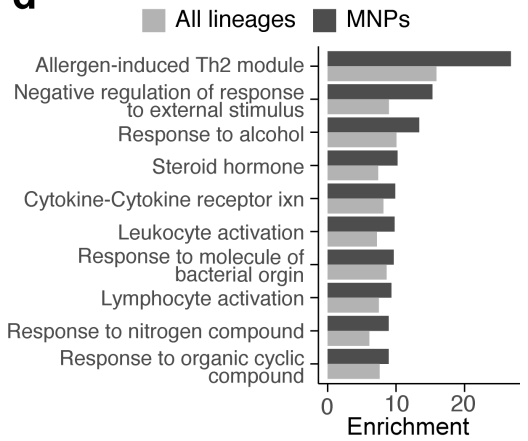

Figure 3. Prioritized genes for IBD

(a) Genes prioritized by ABC-Max for 47 noncoding IBD credible sets linking to 43 unique genes. Heatmap shows $A B C$ scores in 6 biosample categories (maximum value across biosamples within each category). Color scale: quantiles on the $A B C$ score, over the range $[0.015,0.75]$. Blue boxes indicate $\log _{10}$ genomic distance from variant to gene TSS. Black boxes at right indicate if the gene has been implicated in IBD risk based on coding variants or experimental evidence about gene function ${ }^{8}$, is identified by prior eQTL colocalization or TWAS analyses, or is a member of selected gene sets (see Methods). Asterisks mark newly prioritized genes.

(b) Histogram of the distances from the predicted IBD variant to the TSS of the target gene. The two genes with the greatest distances are labeled, along with the cell type of their $\mathrm{ABC}$ predictions.

(c) A comparison of the number of biosample categories (cell type lineages) in which the gene promoter is active versus the number of categories in which a variant is predicted to regulate the gene by ABC-Max.

(d) MSigDB gene sets identified when performing enrichment analysis among the genes prioritized in mononuclear phagocytes (MNPs). The enrichment for a given gene is calculated as the ratio of the frequency at which $\mathrm{ABC}$-predicted genes belong to the gene set, compared to the frequency at which all genes within $1 \mathrm{Mb}$ of these loci belong to the gene set (see Methods). Dark gray bars show enrichment for genes prioritized in MNPs; light gray bars show enrichment among genes prioritized in any lineage. 


\section{ABC links 951 genes to multiple complex traits}

Beyond analysis of loci for a single trait, connecting GWAS variants to target genes across multiple traits could inform the physiological functions of genes and the selection of drug targets ${ }^{53-}$ 55. Accordingly, we used $A B C$ maps to explore the pleiotropy of variant-gene-trait connections across 72 diseases and complex traits, for which ABC-Max identified a total of 4,976 variants that overlapped enhancers linked to 2,249 unique genes (Table S9).

We identified 425 genes that were linked by $A B C$ to the same variant for multiple traits, suggesting how transcriptional regulation of these genes might influence cellular and physiological functions (Fig. 4a-b, Table S11). (105 such genes fit this criterion in the subset of 37 traits with low pairwise genetic correlation, see Methods). For example, OSGIN1 was predicted by ABC to be regulated in hepatocytes by an enhancer that contained rs4782568. This variant has fine-mapping PIP >= $10 \%$ for 14 complex traits including serum levels of proteins and metabolites produced in the liver, including alkaline phosphatase, alanine aminotransferase, albumin, C-reactive protein, ApoB, IGF1, LDL and total cholesterol, and sex hormone binding globulin (Fig. S7a). OSGIN1 encodes a protein that regulates cellular growth in response to oxidative stress ${ }^{56}$, and its transcriptional regulation in the liver may broadly influence hepatic metabolic functions.

We also identified 577 genes that were linked by $A B C$ to different traits through different variants (Fig. 4a-b, Table S11; 288 genes fit this criterion in the subset of 37 traits with low genetic correlation). For each such gene, the predicted variants overlapped $A B C$ enhancers in different sets of biosamples, indicating that cell-type specific transcriptional regulation of these genes may influence different traits. For example, IKZF1 encodes a transcription factor involved in many stages of hematopoietic differentiation, and was linked by $A B C$ to 12 traits via 19 unique variants in 18 credible sets, including variants associated with erythrocyte, monocyte, or neutrophil count that overlapped ABC enhancers in erythroblasts, monocytes, or CD34+ hematopoietic progenitors, respectively (Fig. S7b). Another such gene was MYC, whose expression quantitatively regulates cellular proliferation and which we previously showed has dozens of nearby GWAS associations that overlap putative enhancers ${ }^{57}$. Here, ABC-Max identified connections to 8 traits via fine-mapped variants, including for hematocrit in erythroblasts and lymphocyte count in B and T cells (Table S9).

Interestingly, these 577 genes linked to multiple traits via different variants appeared to have complex enhancer landscapes: they had (i) more predicted ABC enhancer connections (median 466 across all cell types versus 261 for other genes), (ii) more ABC enhancer connections per cell type in which the gene was expressed (median 4.8 versus 3.3) (Fig. 4c), and (iii) more surrounding noncoding sequence (median $301 \mathrm{~Kb}$ versus $128 \mathrm{~Kb}$ distance to the closest neighboring TSSs; this last metric is independent of ABC predictions) (Fig. S7c). Conversely, increased complexity of the regulatory landscape of a gene, as measured by any of these three metrics, correlated with higher odds of being linked to multiple traits via different variants (top decile: odds ratios of 2.4 to 5.5 , Fig. S7d). These observations suggest that genes with complex enhancer landscapes are more likely to influence multiple traits, likely reflecting constraints on their precise cell-type specific transcriptional control ${ }^{58}$. 
a

425 genes:

577 genes:

Multiple traits via one variant Multiple traits via different variants

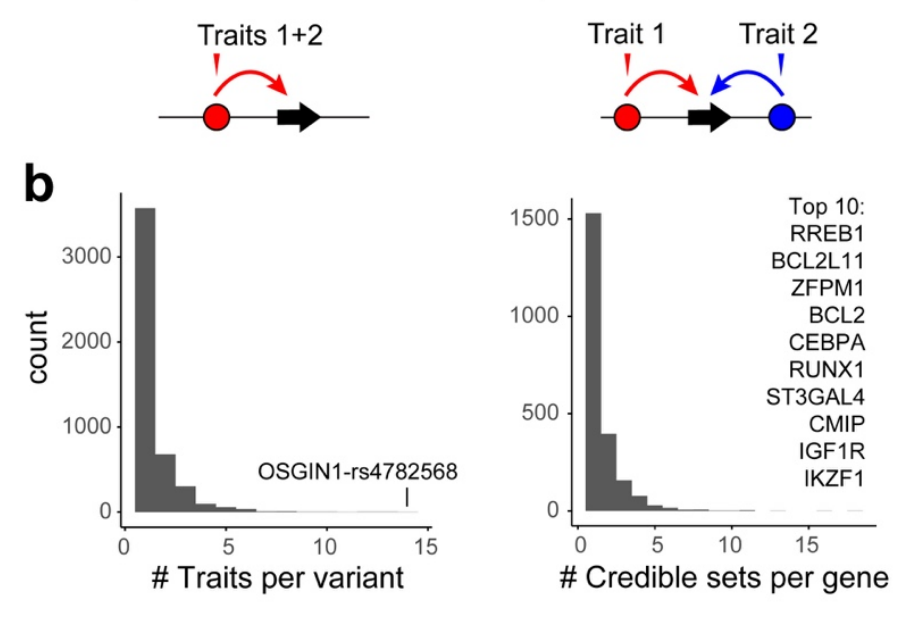

C

\# Traits via different variants: $-0-1-2+$
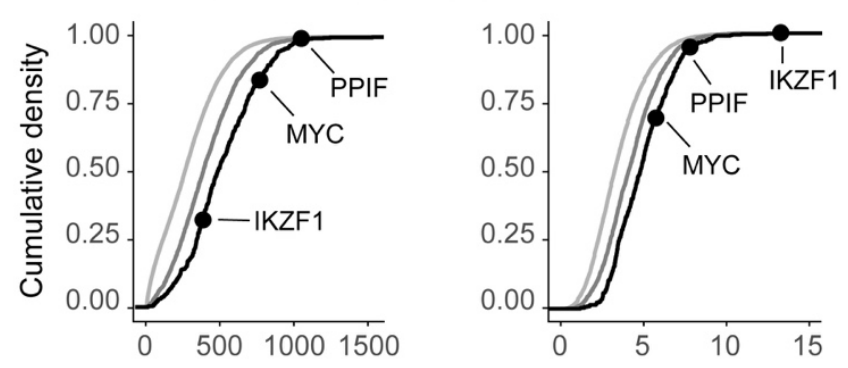

\# Enhancer connections

(all cell types)

\# Enhancer connections (per cell type in which the gene is expressed)

Figure. 4. Pleiotropic effects of variants and genes across 72 diseases and complex traits

(a) Two classes of genes linked by $A B C$ to multiple traits. Circles: enhancers. Black arrows: gene. Colored arrows: $A B C$ predictions. Triangles: variants.

(b) Histograms of properties of $A B C-M a x$ predictions, including the number of linked traits per gene, and number of GWAS signals per gene (unique credible sets with no overlapping variants with PIP $>=10 \%$, see Methods).

(c) Number of predicted enhancer-gene connections for genes linked by ABC to zero traits, one trait by one or more variants, or two or more traits via different variants. Three genes described in text are labeled. 


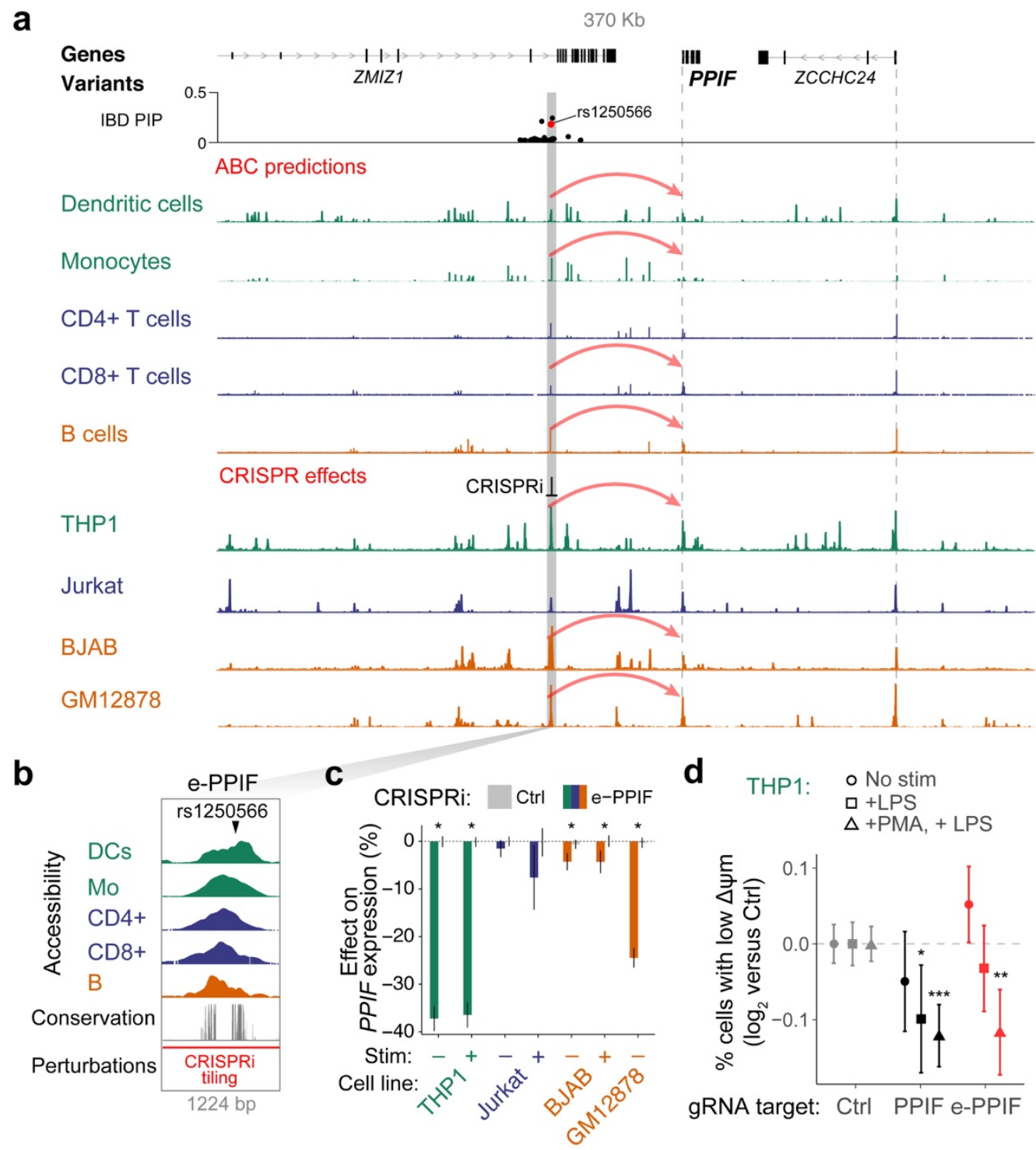

Figure 5. An enhancer regulates PPIF expression and mitochondrial function.

(a) An IBD risk variant (rs1250566) is predicted to regulate PPIF. Signal tracks represent ATAC-seq or DNase-seq. Gray bar highlights enhancer containing rs1250566. Dotted lines mark TSSs. Red arcs at top indicate ABC-Max predictions connecting the enhancer to PPIF. Red arcs at bottom indicate that CRISPRi at the enhancer leads to a significant decrease in expression of PPIF.

(b) 1224-bp region at the PPIF enhancer. Accessibility: DNase- or ATAC-seq from primary immune cell types (DCs=dendritic cells, Mo=monocytes). Conservation: phastCons 100-mammal alignment ${ }^{68}$. Red bar shows the region over which we tiled CRISPRi gRNAs.

(c) Effects of CRISPRi at e-PPIF on the expression of PPIF in human immune cell lines in resting and stimulated (stim) conditions. Error bars show 95\% confidence intervals of the mean. *: two-sided t-test FDR $<0.05$ for 164 CRISPRi gRNAs targeting e-PPIF compared to 814 negative control (Ctrl) gRNAs.

(d) Effects of CRISPRi gRNAs (targeting e-PPIF, PPIF promoter, or negative controls (Ctrl)) on $\Delta \psi_{\mathrm{m}}$, quantified as the frequency of THP1 cells carrying those gRNAs with low versus high MitoTracker Red signal (see Fig. S8a). We tested THP1 cells in unstimulated conditions, stimulated with LPS, and differentiated with phorbol 12-myristate 13-acetate (PMA) and stimulated with LPS (see Methods). Error bars: $95 \%$ confidence intervals for the mean of 40,9 , and 5 gRNAs for Ctrl, PPIF, and e-PPIF, respectively. Two-sided rank-sum $P<0.05\left(^{*}\right),<0.01\left(^{* *}\right)$, or $<0.005\left(^{* *}\right)$ versus Ctrl. 


\section{Linking transcriptional regulation of PPIF to IBD risk}

ABC predictions could accelerate experimental studies to characterize individual GWAS loci, which have been challenging due to the many possible combinations of causal variants, cell types, and target genes ${ }^{1,19}$. To test this, we examined the IBD risk locus at chromosome $10 \mathrm{q} 22.3$, where $A B C$ predictions pointed to an unexpected gene. In this locus, the credible set contains 32 noncoding variants, all of which are located in introns of the gene ZMIZ1. One high-probability variant (rs1250566, PIP $=19 \%$ ) overlapped an ABC enhancer in several immune cell types, including monocytes and dendritic cells (Fig. 5a,b). Interestingly, ABC-Max predicted that the relevant gene was not $Z M I Z 1$, but rather a different nearby gene, PPIF. Although the variant is in an intron of $Z M I Z 1$, PPIF has the higher $A B C$ score because the variant is in more frequent 3D contact with the promoter of PPIF than with the promoter of ZMIZ1 (by a factor of 2.3), likely because the variant is closer to the promoter of PPIF (61 Kb vs $218 \mathrm{~Kb}$, respectively).

PPIF encodes cyclophilin D, a protein expressed in virtually all cell types that regulates metabolism, reactive oxygen species signaling, and cell death via control of the mitochondrial permeability transition pore ${ }^{59-61}$. Ppif ${ }^{/-}$knockout mice are protected from experimental colitis ${ }^{62}$, but PPIF has not been linked to risk for human IBD. PPIF has an extremely complex enhancer landscape: it is in the top $0.3 \%$ of genes with the most $A B C$ enhancer-connections $(1,019$ across all biosamples), the top $2 \%$ with the most enhancers per biosample (average of 7.8 ), and the top $0.3 \%$ of genes with the most genomic sequence covered by its $A B C$ enhancers $(131 \mathrm{~Kb})(\mathbf{F i g}$. 4c). The region around PPIF includes noncoding GWAS signals for 39 other diseases and traits, and fine-mapped variants for these phenotypes overlapped $A B C$ enhancers in cell types including hematopoietic progenitors (for neutrophil count), B cells (for multiple sclerosis and celiac disease), and adipose tissue (for levels of insulin-like growth factor 1) (Fig. S8a). These observations indicate that cell-type specific transcriptional control of PPIF likely influences many complex traits.

To explore the enhancer landscape of PPIF and obtain evidence that variation in the predicted $\mathrm{ABC}$ enhancer could affect IBD risk, we used CRISPRi-FlowFISH ${ }^{24}$ to perturb each of the 163 accessible elements in a $712 \mathrm{~Kb}$ region around PPIF in four human immune cell lines, with and without stimulation with appropriate immune ligands (see Methods). We identified 14 enhancers that regulated PPIF expression in at least one of these cell lines and stimulated states (Fig. S8b, Table S4). The ABC enhancer that contained the fine-mapped IBD variant indeed significantly regulated PPIF expression in resting and activated monocytic (THP1) and B (GM12878, BJAB) cell lines, with the strongest effect in THP1 cells (37\% effect on PPIF expression, two-sided t-test $P<10^{-111}$ ) (Fig. 5c, Fig. S8b-d).

Because PPIF is known to regulate the mitochondrial permeability transition pore and reduce mitochondrial membrane potential $\left(\Delta \psi_{\mathrm{m}}\right)^{59}$, we tested whether this PPIF enhancer might tune $\Delta \psi_{\mathrm{m}}$ in THP1 cells. We infected cells with a pool of CRISPRi gRNAs targeting the PPIF enhancer and promoter, stained cells with MitoTracker Red (a fluorescent dye with higher signal in mitochondria with higher $\Delta \psi_{\mathrm{m}}$ ), sorted cells into 3 bins based on their level of fluorescence, and sequenced the distribution of gRNAs in each bin to infer the effect of each gRNA on $\Delta \psi_{\mathrm{m}}$ (Fig. S8e). CRISPRi targeting of the PPIF enhancer or promoter indeed increased $\Delta \psi_{\mathrm{m}}$ in THP1 cells in LPSstimulated, but not unstimulated, conditions (Fig. 5d, Fig. S8f), consistent with the expected direction of effect of PPIF. These experiments indicate that this enhancer can tune the metabolic state of mitochondria in cells responding to inflammatory stimuli.

Together, these data demonstrate that an enhancer regulates the expression of PPIF to influence mitochondrial function, and implicate transcriptional control of PPIF in genetic risk for IBD. Notably, PPIF is one of the molecular targets of cyclosporine $A(C s A)^{63}$, an immunosuppressive drug used to treat IBD. Although the therapeutic effects of CsA have been thought to involve a different cyclophilin, PPIA, in $\mathrm{T}$ cells ${ }^{64,65}, \mathrm{CsA}$ also inhibits the mitochondrial permeability 
transition $^{59}$ and has been observed to affect the maturation or activation of MNPs in a cell-intrinsic manner ${ }^{66,67}$. Our results highlight the possibility that the immunosuppressive effects of CsA could include inhibition of PPIF.

\section{Discussion}

This work creates genome-wide maps including $>6$ million enhancer-gene connections that illuminate the functions of disease variants. These maps are based on a simple formula that involves multiplying measures of activity and contact, facilitating insights into enhancer function. These ABC enhancer-gene connections can be highly cell-type specific (Fig. S6), consistent with observations that noncoding variants often function in specific cell types and contexts ${ }^{1,19,69}$. Nearly half of enhancers are predicted to regulate multiple genes (Fig. S1d), matching observations from eQTL studies ${ }^{19,47}$. Certain genes are predicted to have up to thousands of enhancer connections across the cell types examined, and their transcriptional regulation in different cell types may influence multiple human diseases and traits (Fig. 4c).

At individual GWAS loci, ABC enhancer maps provide specific, actionable hypotheses linking noncoding variants to cell types, enhancers, and genes. These predictions linked 14 new genes to IBD risk and revealed a role for an enhancer of PPIF in tuning mitochondrial function. We have also prospectively applied $A B C$ maps to identify and validate a variant that regulates $T E T 2$ in hematopoietic progenitors to influence risk for clonal hematopoiesis (Bick et al., in press at Nature $^{70}$ ). By dramatically narrowing the search space of possible variants, cell types, and target genes at any given GWAS locus, ABC maps should accelerate variant-to-function studies for many diseases.

Our study has several limitations that highlight areas for future work (Note S1). (i) Because ABC predictions in a given cell type involve analyzing epigenomic data from a single individual, they will miss enhancers that are present only in certain genotypes or environmental states. Analyzing data across multiple individuals in healthy and disease contexts may help to capture a larger fraction of possible enhancers and interpret additional variants. (ii) While ABC maps appear to do a good job of linking noncoding GWAS variants to target genes, analyzing other complementary data - such as information about gene function - may further improve the ability to identify causal genes for common diseases. (iii) In comparing ABC-Max to other methods for identifying IBD genes (Fig. 2), we note that performance of each approach is a function of both the computational method and the varying cell types included in the dataset (see Methods). Generating deep eQTL maps and enhancer-gene maps in shared systems could help to directly compare various methods at identifying disease genes. (iv) Here we use bulk ATAC-seq and $\mathrm{H} 3 \mathrm{~K} 27$ ac ChIP-seq to create ABC maps. Adapting this approach to leverage single-cell datasets could facilitate expanding these maps across many more cell types.

In summary, our approach illuminates a path toward creating a comprehensive map of enhancer regulation in the human genome. By refining computational models such as $A B C$ and collecting the needed epigenomic data, it should be possible to create an accurate map of enhancers and their target genes in cis across thousands of cell types and states in the human body. These maps would provide a foundational reference for interpreting data from future genome-wide association studies to identify disease genes and cell types. Such a project is becoming feasible, and will be an essential resource for understanding gene regulation and the genetic basis of human diseases. 


\section{Note S1. ABC enhancer-gene maps for interpreting the functions of noncoding GWAS variants}

We set out to develop a method to build accurate enhancer-gene maps that could connect noncoding GWAS signals to target genes. To do so, we designed an approach that could (i) identify causal variants from among the many variants in LD with one another in a GWAS locus, (ii) assess the potential functions of variants on enhancers across many cell types potentially relevant to a disease, (iii) link variants in enhancers to target genes in relevant cell types, and (iv) be generally applicable to many common diseases. In the following sections, we discuss how ABC-Max combines genome-wide ABC maps of enhancer regulation with genetic maps of finemapped GWAS variants to accomplish these goals. We also discuss conceptual differences between $A B C-M a x$ and existing methods for linking noncoding GWAS signals to target genes.

ABC-Max operates on likely causal variants identified by genetic fine-mapping.

A major challenge in leveraging epigenomic predictions to interpret the functions of GWAS variants has been that each GWAS locus typically contains many associated variants in linkage disequilibrium with one another, only one or several of which might be responsible for the association signal. Recent studies have begun to apply genetic fine-mapping methods to prioritize likely causal variants for many diseases and traits ${ }^{20-22}$. Here, we leverage results from two such studies that have applied fine-mapping to IBD and 73 complex traits, using methods that account for the possibility that there are multiple causal signals in a given locus ${ }^{21}$. We analyze variants with individual posterior probabilities above a threshold (>10\% for most analyses), and find that these variants are up to 48-fold enriched in ABC enhancers in particular cell types. Variants above the PIP threshold that overlap ABC enhancers in enriched cell types are highly likely to be causal.

ABC-Max can assess enhancer-gene connections across many cell types.

GWAS variants in enhancers appear to regulate gene expression in specific cell types or contexts ${ }^{1,19,69}$. Therefore, approaches to build regulatory maps to interpret GWAS variants must be able to assess many dozens of cell types potentially relevant to disease risk. To this end, an important feature of the ABC model is that it can make cell-type specific predictions from easily obtainable measurements of chromatin state in a given cell type (minimally, H3K27ac ChIP-seq and either ATAC-seq or DNase-seq; we previously found that cell-type specific measurements of 3D contacts are not required for optimal prediction accuracy, and so we estimate 3D contact frequencies from the average of Hi-C maps in 10 cell types ${ }^{24}$ ). As opposed to eQTL studies, which require measuring RNA expression in hundreds to thousands of individuals, $A B C$ can make predictions based on data from a single individual. These reduced data requirements allow us to build $A B C$ maps across 131 biosamples, leveraging a combination of existing datasets from ENCODE and Roadmap and other new epigenomic datasets that we generated here. With these maps, we can look up the potential effects of a variant across many cell types and states potentially relevant to disease.

ABC-Max links GWAS variants to known disease genes in relevant cell types.

Noncoding GWAS variants might regulate any of the many nearby genes (5-60 within $1 \mathrm{Mb})$ and act in any of the dozens of cell types relevant to disease. To use ABC maps to prioritize among these possibilities, we first identify cell types where fine-mapped variants are globally enriched in enhancers. Second, we link fine-mapped variants that overlap enhancers in enriched cell types to the gene with the highest $A B C$ score. This approach appears to outperform other regulatory methods at identifying genes curated in IBD GWAS loci (Fig. 2).

ABC-Max is generally applicable to many common diseases.

Practically, the ABC model can be applied to many cell types and states in the human body, limited only by the ability to acquire the needed epigenomic datasets. Genetic fine-mapping will be increasingly applied to many common diseases as genetic studies in disease cohorts and 
biobanks expand. As such, we expect that ABC-Max will enable linking thousands of GWAS signals to relevant target genes and cell types to facilitate learning biological mechanisms of disease risk.

Limitations and future directions.

ABC-Max has several limitations that highlight areas for future improvement:

First, ABC-Max does not directly incorporate information about the function or sequence of a single-nucleotide variant, other than its overlap with a predicted enhancer. This affects a number of capabilities. The ABC predictions do not directly inform which transcription factors might be impacted. ABC-Max may miss cases where an activating variant creates an enhancer that does not exist in the input epigenomic datasets (due to the biosamples from which epigenomic data are derived not containing the risk haplotype). Although the $A B C$ model appears to work well for identifying the target genes of fine-mapped GWAS variants (where we have strong evidence from human genetics that a particular variant is functional in some context), further work will be required to assess the extent to which this will help to interpret the functions of rare, de novo, or other variants, such as from whole-genome sequencing studies. These limitations could be addressed by combining ABC-Max with sequence-based models that can infer the effects of single-nucleotide changes on chromatin state ${ }^{71,72}$.

Second, $A B C-M a x$ involves prioritizing the single gene in a locus with the highest $A B C$ score, and does not consider whether other genes also regulated by a variant might contribute to disease risk. Indeed, in a few cases, it appears that genes that do not have the highest ABC score in a locus appear to be relevant to disease (e.g., SORT1 ${ }^{9}$, Table S9), and in several loci there are multiple genes involved in relevant pathways linked to a single variant and enhancer (e.g., TNFSF15 and TNFSF8, Table S8). Future work will explore combining the regulatory predictions of $A B C$ with computational or experimental approaches that consider gene pathways to improve the ability of the model to identify disease genes.

Finally, ABC-Max is designed to predict the functions of variants that affect enhancers, which appear to comprise a majority of noncoding causal GWAS variants. However, some causal noncoding variants likely act through other mechanisms, such as DNA topological elements, RNA splicing, or RNA translation efficiency. Further work will be required to identify and predict the functions of such variants, and to build integrative models that consider all of these mechanisms jointly. 


\section{Methods}

\section{Immune cell lines}

We generated epigenomic data to build the ABC Model and/or performed CRISPRi experiments in the following human immune cell lines: THP1 (monocytic-like cell line, acute monocytic leukemia), BJAB (B cell-like cell line, EBV-negative inguinal Burkitt's lymphoma), GM12878 (EBVimmortalized lymphoblastoid cell line), U937 (monocytic-like cell line, histiocytic lymphoma), and Jurkat ( $T$ cell-like, $T$ cell leukemia).

Cell culture. We maintained cells at a density between $100 \mathrm{~K}$ and $1 \mathrm{M}$ per $\mathrm{ml}(250 \mathrm{~K}-1 \mathrm{M}$ per $\mathrm{ml}$ for GM12878) in RPMI-1640 (Thermo Fisher Scientific, Waltham, MA) with 10\% heat-inactivated FBS (15\% for GM12878, HIFBS, Thermo Fisher Scientific), 2mM L-glutamine, and 100 units $/ \mathrm{ml}$ streptomycin and $100 \mathrm{mg} / \mathrm{ml}$ penicillin by diluting cells $1: 8$ in fresh media every three days.

\section{Stimulation conditions for ABC maps and CRISPRi experiments.}

We stimulated BJAB cells with $4 \mu \mathrm{g} / \mathrm{ml}$ anti-CD40 (Invitrogen-140409-82) and $10 \mu \mathrm{g} / \mathrm{ml}$ anti-lgM (Sigma-I0759) for 4 hours. We stimulated Jurkat cells with $5 \mu \mathrm{g} / \mathrm{ml}$ anti-CD3 (Biolegend-317315) and $100 \mathrm{ng} / \mathrm{ml}$ phorbol 12-myristate 13-acetate (PMA, Sigma-P1585) for 4 hours. We stimulated THP1 cells with $1 \mu \mathrm{g} / \mathrm{ml}$ bacterial lipopolysaccharide (LPS) from E. coli K12 (LPS-EK Invivogen tlrl-peklps) for 4 hours. We stimulated U937 cells with 200 ng/ml LPS for 4 hours.

\section{Stimulation conditions for ABC maps across extended timecourse in THP1 cells}

For THP1 cells, we generated epigenomic data examining a longer time-course, by stimulating with PMA (100 $\mathrm{ng} / \mathrm{mL})$ for 12 hours, then removing PMA and adding LPS $(1 \mu \mathrm{g} / \mathrm{mL})$ and profiling at $0,1,2,6,12,24,48,72,96$, and 120 hours after addition of LPS. Because THP1 cells adhere when stimulated with PMA (changing into a more macrophage-like state), we harvested cells by taking out the media, washing twice, adding TrypLE for 5 minutes at $37^{\circ} \mathrm{C}$, then supplementing with $100 \mu \mathrm{L}$ of media, removing cells from the round-bottom plate and pelleting.

\section{Epigenomic profiling of immune cell lines}

To build $A B C$ maps in human immune cell lines, we generated ATAC-seq and H3K27ac ChIPseq data in BJAB, Jurkat, THP1, and U937 cells, with and without stimulation with the ligands described above.

ATAC-seq. We applied ATAC-seq as previously described ${ }^{73}$, with modifications. Briefly, we washed 50,000 cells once with $50 \mu \mathrm{l}$ of cold 1x PBS and added $50 \mu \mathrm{l}$ of Nuclei Isolation EZ Lysis Buffer (SIGMA NUC101-1KT) to resuspend gently, immediately centrifuging at 500xg for 10 minutes at $4^{\circ} \mathrm{C}$. The lysis buffer was decanted away from the nuclei pellet. Afterwards, we resuspended the nuclei in $100 \mu \mathrm{l}$ of Nuclei Isolation EZ Lysis Buffer again and centrifuged at $500 \times G$ for 5 minutes at $4^{\circ} \mathrm{C}$ and re-decant the lysis buffer, which we found to decrease mitochondrial reads although at the cost of library complexity. We then resuspended the nuclear pellet in $50 \mu \mathrm{l}$ of transposition reaction mix (25 $\mu$ l Buffer TD, $2.5 \mu$ IDE1 (Illumina 15028212); 7.5 $\mu \mathrm{l}$ water, $15 \mu \mathrm{l}$ PBS, to increase salinity which we found to increase signal-to-noise) and incubated the mix at $37^{\circ} \mathrm{C}$ for 30 minutes in a PCR block. Immediately following the transposition reaction, we split the $50 \mu \mathrm{l}$ reaction volume into two and we added $25 \mu \mathrm{l}$ of guanidine hydrochloride (Buffer PB, Qiagen 28606) to each as a chaotropic agent to stop the reaction and dissociate the proteins and transposase from the DNA. Keeping one of the reactions as backup, we proceeded with one by adding 1.8X SPRI beads (Agencourt A63881), waiting 5 minutes for the DNA to associate to the beads, and then washing the beads twice using $80 \% \mathrm{EtOH}$. We then eluted the DNA from the 
beads using $10 \mu \mathrm{l}$ of water and added to it $25 \mu \mathrm{l}$ NEBNext HiFi 2x PCR MasterMix (NEB M0541), with $2.5 \mathrm{uL}$ of each of the dual-indexed Illumina Nextera primers $(25 \mu \mathrm{M})$. We amplified the PCR reaction to 15 cycles, as previously described. We purified amplified libraries and removed adapters using two clean-ups with 1.8x volume SPRI (Agencourt A63881). We sequenced these libraries on an Illumina HiSeq 2500. We filtered, aligned, and processed the data to generate BAM files as previously described ${ }^{48}$.

H3K27ac ChIP-seq. We generated and analyzed ChIP-seq data from 5 million cells in each cell line and stimulation state, following protocols previously described ${ }^{74}$. Before harvesting for ChIPseq, cells at 1 million cells per $\mathrm{mL}$ were replenished by a 1:2(v/v) split in fresh media and allowed to grow for 4 hours. 10 million cells were harvested from each cell type at $500 \mathrm{~K}$ cells $/ \mathrm{mL}$ and washed $2 x$ in cold PBS. Cells were resuspended in warm PBS with $1 \%$ formaldehyde (Cat \#28906, Thermo Scientific) and incubated at $37^{\circ} \mathrm{C}$ for 10 minutes. Crosslinking was quenched by adding glycine to a concentration of $250 \mathrm{mM}$ and incubating for 5 minutes at $37^{\circ} \mathrm{C}$. Cells were placed on ice for 5 minutes, then washed $2 x$ in ice-cold PBS and snap-frozen in liquid nitrogen and stored. Later, crosslinked cells were lysed in $1 \mathrm{~mL}$ cell lysis buffer $(20 \mathrm{mM}$ Tris $\mathrm{pH}$ 8.0, 85 $\mathrm{mM} \mathrm{KCl}, 0.5 \% \mathrm{NP} 40$ ) and incubated on ice for 10 minutes. The nuclear pellet was isolated by spinning the cell lysis mix at $5,600 \mathrm{xg}$ at $4^{\circ} \mathrm{C}$ for 3.5 minutes and discarding the supernatant. Nuclear pellets were lysed by adding $1 \mathrm{~mL}$ nuclear lysis buffer $(10 \mathrm{mM} \mathrm{Tris}-\mathrm{HCl} \mathrm{pH} 7.5 \mathrm{ml}, 1 \%$ NP-40 alternative (CAS 9016-45-9), 0.5\% Na Deoxycholate, 0.1\% SDS) with protease inhibitors on ice for 10 minutes. The chromatin-containing nuclear lysate was sonicated $3 x$ using a Branson sonifier (ON 0.7s, OFF 1.3s, TIME 2 minutes, WATTS 10-12), with 1 minute rest between sonifications. Sonicated chromatin was spun down at maximum speed. $300 \mu \mathrm{L}$ of the clarified supernatant was diluted 1:1 with ChIP dilution buffer $(16.7 \mathrm{mM}$ Tris- $\mathrm{HCl} \mathrm{pH} 8.1,1.1 \%$ Triton X100 , and $167 \mathrm{mM} \mathrm{NaCl}, 1.2 \mathrm{mM}$ EDTA, $0.01 \%$ SDS). To immunoprecipitate H3K27ac, $3 \mu \mathrm{l}$ of H3K27ac monoclonal antibody (Cat \#39685, Active Motif) was added to each sample and rotated overnight at $4^{\circ} \mathrm{C}$. The following morning, 50 uL of a 1:1 mix of Protein A (Cat \#10008D, Invitrogen) and Protein G Dynabeads magnetic beads (Cat \#10004D, Life Technologies) were washed with blocking buffer (PBS, 0.5\% Tween20, 0.5\% BSA with protease inhibitors), resuspended in $100 \mu \mathrm{l}$ blocking buffer, and added to each sample. The samples were rotated end-over-end for $1 \mathrm{~h}$ at $4^{\circ} \mathrm{C}$ to capture antibody complexes, then washed as follows: once with $200 \mu \mathrm{l}$ Low-Salt RIPA buffer $(0.1 \%$ SDS, 1\% Triton X-100, $1 \mathrm{mM}$ EDTA, $20 \mathrm{mM}$ Tris- $\mathrm{HCl} \mathrm{pH} 8.1,140 \mathrm{mM} \mathrm{NaCl}, 0.1 \%$ $\mathrm{Na}$ Deoxycholate), once with $200 \mu \mathrm{L}$ High-Salt RIPA buffer $(0.1 \%$ SDS, $1 \%$ Triton X-100, $1 \mathrm{mM}$ EDTA, $20 \mathrm{mM}$ Tris-HCl pH 8.1, $500 \mathrm{mM} \mathrm{NaCl}, 0.1 \% \mathrm{Na}$ Deoxycholate), twice with $200 \mu \mathrm{L} \mathrm{LiCl}$ buffer (250 mM LiCl, 0.5\% NP40, 0.5\% Na Deoxycholate, 1 mM EDTA, 10 mM Tris-HCl pH 8.1), and twice with $200 \mu \mathrm{l} \mathrm{TE}$ buffer $(10 \mathrm{mM}$ Tris- $\mathrm{HCl} \mathrm{pH} \mathrm{8.0,1} \mathrm{mM} \mathrm{EDTA} \mathrm{pH} \mathrm{8.0).} \mathrm{Chromatin} \mathrm{was}$ then eluted from the beads with $60 \mu \mathrm{l} \mathrm{ChIP} \mathrm{elution} \mathrm{buffer}(10 \mathrm{mM}$ Tris-HCl pH 8.0, $5 \mathrm{mM}$ EDTA, $300 \mathrm{mM} \mathrm{NaCl}, 0.1 \%$ SDS). Crosslinking was reversed by adding $8 \mu \mathrm{L}$ of reverse cross-linking enzyme mix (250 mM Tris-HCl pH 6.5, $62.5 \mathrm{mM}$ EDTA pH 8.0, $1.25 \mathrm{M} \mathrm{NaCl}, 5 \mathrm{mg} / \mathrm{ml}$ Proteinase K (Cat \#25530-049, Invitrogen), $62.5 \mu \mathrm{g} / \mathrm{ml}$ RNase A (Cat \#111199150001, Roche)) to each immunoprecipitated sample, as well as to $10 \mu \mathrm{l}$ of the sheared chromatin input for each sample brought to volume of $60 \mu \mathrm{l}$ ChIP elution buffer. Reverse crosslinking reactions were incubated 2 $\mathrm{h}$ at $65^{\circ} \mathrm{C}$ and cleaned using Agencourt Ampure XP SPRI beads (Cat \#A63880, Beckman Coulter) with a $2 x$ bead:sample ratio. Sequencing libraries were prepared with KAPA Library Preparation kit (Cat \#KK8202, KAPA Biosystems). ChIP libraries were sequenced using single-end sequencing on an Illumina Hiseq 2500 machine (Read 1: 76 cycles, Index 1: 8 cycles), to a depth of $>30$ million reads per ChIP sample.

\section{Curation of published epigenomic data}

Table S2 lists the data sources for each ABC biosample, and Table S1 describes the epigenomic datasets generated for this study. 
ENCODE. We downloaded BAM files for DNase-seq and H3K27ac ChIP-seq experiments from the ENCODE Portal on July $17,2017^{75}$. We selected hg19-aligned BAM files that were marked as "released" by the ENCODE Portal and were not flagged as "unfiltered", "extremely low spot score", "extremely low read depth", "NOT COMPLIANT", or "insufficient read depth".

Roadmap. We downloaded BAM files for DNase-seq and H3K27ac ChIP-seq from the Roadmap Epigenomics Project (http://egg2.wustl.edu/roadmap/data/byFileType/alignments/consolidated/) on July $12,2017^{45}$.

Other studies. We downloaded FASTQ files for DNase-seq, ATAC-seq, and ChIP-seq data from 13 other studies (Table S2), and processed them using our custom pipelines as described below.

Merging cell types. We created a list of cell types across all sources for which we had at least one chromatin accessibility experiment (DNase-seq or ATAC-seq) and one H3K27ac ChIP-seq experiment. In cases where the same cell types were included in data from the Roadmap Epigenome Project and also from the ENCODE Portal, we used the processed data from Roadmap. In some cases, we combined data from multiple sources (e.g., ENCODE data and our own datasets) to expand the number of cell types considered. As a result of this merging, some "cell types" in our dataset represent data from a single donor and experimental sample, whereas others involve a mixture of multiple donors and/or experimental samples.

\section{Processing of ATAC-seq and ChIP-seq data}

We aligned reads using BWA (v0.7.17) ${ }^{76}$, removed PCR duplicates using the MarkDuplicates function from Picard (v1.731, http://picard.sourceforge.net), and filtered to uniquely aligning reads using samtools (MAPQ >= 30, https://github.com/samtools/samtools) $)^{77}$. The resulting BAM files were used as inputs into the $\mathrm{ABC}$ Model.

\section{Activity-by-Contact model predictions}

We used the Activity-by-Contact (ABC) model (https://github.com/broadinstitute/ABC-EnhancerGene-Prediction) to predict enhancer-gene connections in each cell type, based on measurements of chromatin accessibility (ATAC-seq or DNase-seq) and histone modifications (H3K27ac ChIP-seq), as previously described ${ }^{24}$. In a given cell type, the ABC model reports an "ABC score" for each element-gene pair, where the element is within $5 \mathrm{Mb}$ of the TSS of the gene.

Briefly, for each cell type, we:

1. Called peaks on the chromatin accessibility dataset using MACS2 with a lenient $p$-value cutoff of 0.1

2. Counted chromatin accessibility reads in each peak and retained the top 150,000 peaks with the most read counts. We then resized each of these peaks to be $500 \mathrm{bp}$ centered on the peak summit. To this list we added $500 \mathrm{bp}$ regions centered on all gene TSS's and removed any peaks overlapping blacklisted regions (version 1 from https://sites.google.com/site/anshulkundaje/projects/blacklists) ${ }^{15,78}$. Any resulting overlapping peaks were merged. We call the resulting peak set candidate elements.

3. Calculated element Activity as the geometric mean of quantile normalized chromatin accessibility and H3K27ac ChIP-seq counts in each candidate element region. Chromatin accessibility and H3K27ac ChIP-seq signals in each candidate element were quantile normalized to the distribution observed in K562 cells. 
4. Calculated element-promoter Contact using the average $\mathrm{Hi}-\mathrm{C}$ signal across 10 human $\mathrm{Hi}-$ C datasets as described below.

5. Computed the ABC Score for each element-gene pair as the product of Activity and Contact, normalized by the product of Activity and Contact for all other elements within 5 $\mathrm{Mb}$ of that gene.

Average Hi-C

To generate a genome-wide averaged $\mathrm{Hi}-\mathrm{C}$ dataset, we downloaded $\mathrm{KR}$ normalized $\mathrm{Hi}-\mathrm{C}$ matrices for 10 human cell types ${ }^{24}$. For each cell type we:

1. Transformed the Hi-C matrix for each chromosome to be doubly stochastic.

2. We then replaced the entries on the diagonal of the Hi-C matrix with the maximum of its four neighboring bins.

3. We then replaced all entries of the $\mathrm{Hi}-\mathrm{C}$ matrix with a value of $\mathrm{NaN}$ or corresponding to $\mathrm{KR}$ normalization factors $<0.25$ with the expected contact under the power-law distribution in the cell type.

4. We then scaled the Hi-C signal for each cell type using the power-law distribution in that cell type as previously described.

5. We then computed the "average" Hi-C matrix as the arithmetic mean of the 10 cell-type specific Hi-C matrices. This $\mathrm{Hi}-\mathrm{C}$ matrix (5 $\mathrm{Kb}$ resolution) is available here: $\mathrm{ftp}: / /$ ftp.broadinstitute.org/outgoing/lincRNA/average hic/average hic.v2.191020.tar.gz

\section{Estimating promoter activity}

In each cell type, we assign enhancers only to genes whose promoters are "active" (i.e., where the gene is expressed and that promoter drives its expression). We defined active promoters as those in the top $60 \%$ of Activity (geometric mean of chromatin accessibility and H3K27ac ChIPseq counts). We used the following set of TSSs (one per gene symbol) for ABC predictions, as previously described ${ }^{24}$ : https://github.com/broadinstitute/ABC-Enhancer-GenePrediction/blob/v0.2.1/reference/RefSeqCurated.170308.bed.CollapsedGeneBounds.bed. We note that this approach does not account for cases where genes have multiple TSSs either in the same cell type or in different cell types.

For computing global statistics of $A B C$ enhancer-gene connections (Fig. S1), we considered all distal element-gene connections with an $\mathrm{ABC}$ score $>=0.015$ and within a distance of $2 \mathrm{Mb}$.

\section{Processing $A B C$ predictions for variant overlaps}

For intersecting $A B C$ predictions with variants, we took the predictions from the $A B C$ Model and applied the following additional processing steps: (i) We considered all distal element-gene connections with an ABC score >= 0.015 (see Fig. S3; lower threshold than our previous study ${ }^{24}$ to increase recall and identify gain-of-function variants that increase enhancer activity), and all distal or proximal promoter-gene connections with an $A B C$ score $>=0.1$ (based on our previous experimental data ${ }^{24}$ ). (ii) We shrunk the $\sim 500$-bp regions by 150 -bp on either side, resulting in a $\sim 200$-bp region centered on the summit of the accessibility peak. This is because, while the larger region is important for counting reads in H3K27ac ChIP-seq, which occur on flanking nucleosomes, DNA sequences important for enhancer function are likely located in the central nucleosome-free region. (iii) We included enhancer-gene connections spanning up to $2 \mathrm{Mb}$.

\section{CRISPRi-FlowFISH}


We applied CRISPRi-FlowFISH to very sensitively test the effects of distal elements on gene expression $^{24}$. Briefly, CRISPRi-FlowFISH involves targeting putative enhancers with many independent guide RNAs (gRNAs; median $=45$ ) in a pooled screen using CRISPR interference (CRISPRi), which alters chromatin state via recruitment of catalytically dead Cas 9 fused to a KRAB effector domain. After infecting a population of cells with a gRNA lentiviral library, we estimate the expression of a gene of interest. Specifically, we: (i) use fluorescence in situ hybridization (FISH, Affymetrix PrimeFlow assay) to quantitatively label single cells according to their expression of an RNA of interest; (ii) sort labeled cells with fluorescence-activated cell sorting (FACS) into 6 bins based on RNA expression; (iii) use high-throughput sequencing to determine the frequency of gRNAs from each bin; and (iv) compare the relative abundance of gRNAs in each bin to compute the effects of gRNAs on RNA expression. CRISPRi-FlowFISH provides $\sim 300$ bp resolution to identify regulatory elements; has power to detect effects of as low as $10 \%$ on gene expression; and provides effect size estimates that match those observed in genetic deletion experiments ${ }^{24}$.

Here, we applied CRISPRi-FlowFISH to comprehensively test all putative enhancers in a $\sim 700$ $\mathrm{Kb}$ region around PPIF, and to validate additional selected enhancers (for 12 additional genes) that contained variants associated with IBD or other immune diseases or traits. For CRISPRiFlowFISH experiments for PPIF, we designed gRNAs tiling across all accessible regions (here, defined as the union of the MACS2 narrow peaks and 250-bp regions on either side of the MACS2 summit) in the range chr10:80695001-81407220 in any of the following cell lines (+/- stimulation as described above): THP1, BJAB, Jurkat, GM12878, K562, Karpas-422, or U937. For CRISPRiFlowFISH experiments for other genes, we included gRNAs targeting the promoter of the predicted gene and selected enhancer(s) nearby. We excluded gRNAs with low specificity scores or low-complexity sequences as previously described ${ }^{24}$. We generated cell lines expressing KRAB-dCas9-IRES-BFP under the control of a doxycycline-inducible promoter (Addgene \#85449) and the reverse tetracycline transactivator (rtTA) and a neomycin resistance gene under the control of an EF1 $\alpha$ promoter (ClonTech, Mountain View, CA), as previously described ${ }^{57}$. For each, we sorted polyclonal populations with high BFP expression upon addition of doxycycline. For GM12878 cells, we used an alternative lentiviral construct to express the rtTA with a hygromycin resistance gene, as GM12878 appeared resistant to selection with neomycin/G418.

We performed CRISPRi-FlowFISH using ThermoFisher PrimeFlow (ThermoFisher 88-18005210) as previously described, using the probesets listed in Table 1. To ensure robust data, we only included probesets with twofold signal over unstained cells, and required an uncorrected knockdown at the TSS of $>20 \%$. We analyzed these data as previously described ${ }^{24}$. Briefly, we counted gRNAs in each bin using Bowtie ${ }^{79}$ to map reads to a custom index, normalized gRNA counts in each bin by library size, then used a maximum-likelihood estimation approach to compute the effect size for each gRNA. We used the limited-memory Broyden-Fletcher-GoldfarbShanno algorithm (implemented in the $\mathrm{R}$ stats4 package) to estimate the most likely log-normal distribution that would have produced the observed guide counts, and the effect size for each gRNA is the mean of its log-normal fit divided by the average of the means from all negativecontrol gRNAs. As previously described, we scaled the effect size of each gRNA in a screen linearly so that the strongest 20 -guide window at the TSS of the target gene has an $85 \%$ effect, in order to account for non-specific probe binding in the RNA FISH assay (this is based on our observation that promoter CRISPRi typically shows $80-90 \%$ knockdown by $q P C R)^{24}$. We averaged effect sizes of each gRNA across replicates and computed the effect size of an element as the average of all gRNAs targeting that element. We assessed significance using a two-sided t-test comparing the mean effect size of all gRNAs in a candidate element to all negative-control guides. We computed the FDR for elements using the Benjamini-Hochberg procedure and used an FDR threshold of 0.05 to call significant regulatory effects. 


\begin{tabular}{|l|l|}
\hline Gene for FlowFISH & PrimeFlow Assay ID \\
\hline BLK & VA1-3001817-PF \\
\hline ETS1 & VA1-3000680-PF \\
\hline ICOSLG & VA1-16627-PF \\
\hline IFNGR2 & VA1-3003062-PF \\
\hline ITGAL & VA1-3003135-PF \\
\hline CD83 & VA1-3005784-PF \\
\hline PFKFB3 & VA1-3003817-PF \\
\hline IL10 & VA1-10840-PF \\
\hline SEPHS2 & VA1-3007219-PF \\
\hline CD40 & VA1-18558-PF \\
\hline IL6ST & VA1-14473-PF \\
\hline PPIF & VA1-3000735 \\
\hline IL2RA & VA1-3000167-PF \\
\hline
\end{tabular}

\section{Table 1. FlowFISH Probesets}

\section{Comparison of $\mathrm{ABC}$ predictions to genetic perturbations}

We evaluated the ability of the ABC Score and other enhancer-gene prediction methods to predict the results of genetic perturbations using a precision recall framework. For this analysis the true positives are the experimentally measured element-gene pairs which are statistically significant and for which perturbation of the element resulted in a decrease in gene expression. For these comparisons, (i) we only considered experimentally tested elements in which the element is not within 500bp of an annotated gene transcription start site; (ii) for perturbations using CRISPRi we excluded pairs in which the element resides within the gene body of the assayed gene; (iii) we excluded non-significant pairs for which the power to detect a $25 \%$ change in gene expression was < 80\%; and (iv) we only included pairs for which the gene is protein-coding (although the ABC model can make predictions for non-coding genes, many of the other predictions methods we compare to do not make predictions for such genes).

For each experimentally measured element-gene-cell-type tuple, we intersected this tuple with the tuple in the predictions database corresponding to the same cell type, same gene and overlapping element. In cases in which the genomic bounds of an experimentally tested element overlap multiple predicted elements, we aggregated the prediction scores using an aggregation metric appropriate to each individual predictor (for ABC we used 'sum', for correlation- or confidence-based predictors we used 'max'). Similarly, if the predictor did not make a prediction for a particular tuple, it received an arbitrary quantitative score less than the least confident score for the predictor (for $A B C$ we used 0 , for other predictors we used $0,-1,1$ as appropriate). Table S5 lists the experimental data merged with the predictions.

In the cases in which an enhancer-gene prediction method did not make cell-type specific predictions, we evaluated the predictions against experimental data in all cell types (Fig. S3b). We calculated the area under the precision-recall curve (AUPRC) for predictors, or, if the predictor was defined at only one point, we multiplied the precision by the recall.

\section{Similarity of ABC Predictions among replicates and biosamples}

We evaluated the reproducibility of $A B C$ predictions derived from replicate epigenetic experiments. For each biosample in which independent biological replicate experiments for both ATAC-Seq (or DNase-Seq) or H3K27ac ChIP-Seq were available, we generated ABC predictions for replicates 1 and 2 separately. In order to facilitate the reproducibility analysis, when computing 
the ABC Scores for replicate 2, we used the candidate enhancer regions from replicate 1. (Using different sets of candidate regions can confound computing reproducibility. For example, the procedure to define candidate regions (peak calling, extending and merging) could call two separate $\sim 500 \mathrm{bp}$ regions in one replicate, but merge them into a $\sim 1-\mathrm{kb}$ region in the second replicate due to minor differences in the peak summits between replicates. In such a case the $A B C$ Score of the $\sim 1-k b$ region would be equal to the sum of the $A B C$ Scores of the 500-bp regions.)

We then evaluated the quantitative reproducibility of the predictions (Fig. S2b) and the number of predictions shared between replicates (Fig. S2c). We observed that on average $85 \%$ of enhancer-gene predictions in one replicate are shared in the other replicate (at an ABC Score threshold of 0.015). The fraction of shared connections between biological replicates increased as the ABC score cutoff increased: $95 \%$ of connections called in replicate 1 at a higher confidence threshold of 0.02 were also called in replicate 2 (at the default threshold of 0.015 ).

We also evaluated the extent to which the reproducibility of $A B C$ predictions depends on the reproducibility of the underlying epigenetic data. For each biosample, we computed the correlation between the ATAC-Seq (or Dnase-Seq) or H3K27ac ChIP-Seq signals in the candidate regions for that biosample. As expected, we observed that the fraction of shared ABC predictions between replicates increased as the correlation of the underlying epigenetic data increased (Fig. S2d).

We used a similar calculation to compare $A B C$ predictions across cell types and biosamples. For each pair of biosamples we computed the fraction of predicted enhancer-gene connections shared between the pair. For this analysis we used the shrunken ABC elements ( 200bp, see above) and considered two connections to be shared if the elements overlapped at least $1 \mathrm{bp}$ and predicted to regulate the same gene.

\section{Genetic data and fine-mapping}

We downloaded summary statistics for IBD, Crohn's disease (CD), and ulcerative colitis (UC) (European ancestry only) ${ }^{80}$ from https://www.ibdgenetics.org/downloads.html. We obtained finemapping posterior probabilities and credible sets from Huang et al. ${ }^{21}$, and analyzed the top two conditionally independent credible sets in each locus. We also analyzed variants from IBD GWAS loci that were not fine-mapped in this study49,80; for each such locus, we analyzed all 1000 Genomes variants in LD with the lead variant $\left(r^{2}>0.2\right)$ and weighted each variant evenly (probability $=1$ / number of variants in LD). We observed similar results for cell type enrichments with or without including these non-fine-mapped sets. Throughout this text, analyses of "IBD" signals are defined as signals associated with CD, UC, or both.

We obtained fine-mapping results and summary statistics for 73 other traits based on an unpublished analysis (Jacob Ulirsch, Masahiro Kanai, and Hilary Finucane) that analyzed data from the UK Biobank (Application \#31063; fine-mapping available at https://www.finucanelab.org/data). In this analysis, fine-mapping was performed using the Sum of Single Effects (SuSiE) method ${ }^{81}$, allowing for up to 10 causal variants in each region. Prior variance and residual variance were estimated using the default options, and single effects (potential 95\% CSs) were pruned using the standard purity filter such that no pair of variants in a CS could have $r^{2}>0.25$. Regions were defined for each trait as $+/-1.5 \mathrm{Mb}$ around the most significantly associated variant, and overlapping regions were merged. As inputs to SuSiE, summary statistics for each region were obtained using BOLT-LMM ${ }^{82}$ for quantitative traits and SAIGE ${ }^{83}$ for binary traits, in sample dosage LD was computed using LDStore $^{84}$, and phenotypic variance was computed empirically. Variants in the MHC region (chr6: 25-36 Mb) were excluded 
as were $95 \%$ CSs containing variants with fewer than 100 minor allele counts. Coding (missense and predicted loss of function) variants were annotated using the Variant Effect Predictor (VEP) version $85^{85}$. For analysis with $A B C$, we excluded neuropsychiatric traits (for which we expect existing enhancer-gene maps will not include the appropriate cell types), and analyzed only the variants that SuSIE assigned to belong to $95 \%$ credible sets (cs_id != -1).

For all traits, except where specified, we considered only the "noncoding credible sets" — i.e., those that did not contain any variant in a coding sequence or within $10 \mathrm{bp}$ of a splice site annotated in the RefGene database (downloaded from UCSC Genome Browser on 24/06/2017) ${ }^{86}$. We note that predictions for all credible sets, both coding and noncoding, are reported in Table S9 to facilitate future analyses.

\section{Defining enriched biosamples for each trait}

For a given trait, we intersected variants with PIP $>=10 \%$ in noncoding credible sets with $\mathrm{ABC}$ enhancers (or other genomic annotations). For each biosample, we calculated a $P$-value using a binomial test comparing the fraction at which PIP $>=10 \%$ variants overlapped $A B C$ enhancers with the fraction at which all common variants overlap $A B C$ enhancers in that cell type. We calculated the latter using common variants in 1000 Genomes as described in the S-LDSC section. For each trait, we defined a biosample as significantly enriched for that trait if the Bonferroni-corrected binomial $P$-value was $<0.001$.

\section{Stratified linkage disequilibrium score regression (S-LDSC)}

We used S-LDSC to assess the enrichment of disease or trait heritability in ABC enhancers, considering all variants across the genome ${ }^{87}$. We analyzed the $A B C$ enhancer regions as defined above, and ran LD score regression using the baselineLD_v1.1 model using the 1000G_EUR_Phase3_baseline file (downloaded from https://data.broadinstitute.org/alkesgroup/LDSCORE/). For comparison, we also analyzed heritability enrichment in all other accessible regions for each trait. Specifically, we took the list of MACS2 peaks (FDR < 0.05), removed those that overlapped ABC enhancers, and used these regions in S-LDSC.

\section{Partitioning the genome into disjoint functional categories}

To compare the frequency of variants occurring in $A B C$ enhancers as opposed to other functional elements such as coding sequences and splice sites (Fig. S4c), we partitioned the genome into the following functional categories, using the RefGene database (downloaded from UCSC Genome Browser on 24/06/2017): coding sequences (CDS), 5' and 3' untranslated regions (UTR) of protein-coding genes, splice sites (within $10 \mathrm{bp}$ of a intron-exon junction of a protein-coding gene) of protein-coding genes, promoters ( $\pm 250 \mathrm{bp}$ from the gene TSS) of protein-coding genes, $A B C$ enhancers in 131 biosamples, other accessible regions in the same biosamples not called as $A B C$ enhancers, and other intronic or intergenic regions. These categories may overlap; a disjoint annotation was created by assigning each nucleotide to the first of any overlapping categories in the order above (e.g., nucleotides in both coding sequences and $A B C$ enhancers were counted as coding sequences).

\section{Evaluating gene prediction methods}


Curated genes for inflammatory bowel disease. We analyzed a list of IBD disease genes curated by Graham and Xavier (2020). ${ }^{8}$ To evaluate methods to connect noncoding GWAS variants to genes, we analyzed credible sets within $1 \mathrm{Mb}$ of exactly 1 of these known genes that did not contain any protein-coding or splice site variants. In cases where the gene was curated based on evidence from coding variation, we examined nearby conditionally independent noncoding signals, which might act via regulatory effects on the same gene that carries the coding variant.

Gene set enrichment for IBD predictions. As a second approach for comparing methods for identifying causal genes in IBD GWAS loci, we examined the extent to which the predicted genes were enriched for any gene sets. To do so, we downloaded curated and Gene Ontology gene sets from the Molecular Signatures Database ${ }^{46}$. We analyzed all 93 noncoding IBD credible sets. For each gene set, we tested whether it was enriched in the genes predicted by a given method, using the set of all genes within $1 \mathrm{Mb}$ of IBD credible sets as the background, excluding HLA genes. For Fig. $\mathbf{2 b}$, we applied this approach to each of the methods described in Fig. 2a, selected the 5 gene sets with the highest enrichment that also had at least five identified genes and hypergeometric test $P$-value $<10^{-4}$. We plotted a CDF of the enrichments for each of the methods across the union of the top 5 gene sets identified by any of the methods.

\section{Comparisons to alternative variant-to-gene prediction methods}

We compared $A B C-M a x$ to alternative methods to link regulatory variants to disease genes.

eQTL Colocalization (Open Targets Platform). OpenTargets.org performed colocalization analysis between IBD GWAS signals ${ }^{49,80}$ and eQTLs and pQTLs using coloc. This analysis involved QTL datasets from a variety of sources including dozens of human tissues and many immune cell types. We downloaded colocalization results from ftp://ftp.ebi.ac.uk/pub/databases/opentargets/genetics/190505/v2d coloc/ on February 1, 2020, and defined two gene sets: genes showing colocalization with an eQTL or pQTL in an immune cell type or spleen, blood, or gut tissue; and genes showing colocalization with an eQTL or pQTL in any biosample. We considered genes with coloc h4 probability $>=0.9$, and h4/h3 ratio $>=2$. We used the coloc h4 probability to rank genes within each locus.

eQTL Colocalization (JLIM). Chun et al. tested colocalization of IBD GWAS signals with eQTLs in CD4+ T cells, CD14+ monocyte, and LCLs ${ }^{35}$. We obtained their colocalized genes from Table 2. We used the JLIM p-value to rank genes within each locus.

TWAS (S-PrediXcan and multiXcan). Barbeira et al. developed multiXcan and compared GTEx v7 eQTLs to IBD summary statistics ${ }^{32}$. We downloaded Dataset 6 and compared genes within each locus using the multiXcan $p$-value.

Mendelian randomization. Hauberg et al. used a Mendelian randomization based approach (HEIDI) to connect IBD GWAS signals to effects on gene expression using eQTL data from 24 tissues $^{37}$. We downloaded Table S3 and defined predicted genes using either all tissues or those observed in whole blood. We used the SMR false discovery rate to rank genes within each locus.

COGS. Javierre et al. (2016) used promoter-capture Hi-C data in many blood cell types to link GWAS variants to target genes ${ }^{88}$. We downloaded Table S3 (Tab 2) and analyzed genes linked with COGS scores $>=0.5$.

In all cases, we combined predictions of disease genes for IBD, UC, and CD. 


\section{Comparisons to previous enhancer-gene predictions}

We compared the ABC model to methods using alternative enhancer-gene linking approaches. For each of the methods below, we downloaded previous predictions of enhancer-gene links, and assessed (i) their ability to predict enhancer-gene regulation in CRISPR datasets (Fig. S3) and (ii) their ability to identify IBD genes (Fig. 2). For the latter analysis, we used the predictions from each method to overlap fine-mapped variants (PIP $>=10 \%$ ) with enhancers in any cell type and assigned variants to the predicted gene(s).

Promoter-capture Hi-C. We downloaded Data S1 peak data from Javierre et al. (2016) representing promoter-capture $\mathrm{Hi}-\mathrm{C}$ data from 9 hematopoietic cell types, and selected the promoter-distal region pairs with $\mathrm{CHiCAGO}$ score $>=5$. For comparison to CRISPR data we used the $\mathrm{CHiCAGO}$ score as a quantitative predictor.

DHS-promoter correlation (ENCODE2). Thurman et al. (2012) linked distal accessible elements with gene promoters by looking at correlation of DNase I hypersensitivity across 125 cell and tissue types from $\mathrm{ENCODE}^{16}$. We downloaded these links from $\mathrm{ftp}$ ://ftp.ebi.ac.uk/pub/databases/ensembl/encode/integration_data_jan2011/byDataType/openc hrom/jan2011/dhs_gene_connectivity/genomewideCorrs_above0.7_promoterPlusMinus500kb_ withGeneNames_32celltypeCategories.bed8.gz. GWAS loci with high-confidence fine-mappēd variants that overlapped these regions were assigned to the linked gene(s).

eRNA-mRNA correlation (FANTOM5). Andersson et al. (2014) linked transcriptional activity of enhancer and transcription start sites using the FANTOM5 CAGE expression atlas ${ }^{40}$. We downloaded these predictions from http://enhancer.binf.ku.dk/presets/enhancer tss associations.bed.

Enhancer-gene correlation (Ernst Roadmap). Liu, Ernst et al. (2017) correlated gene expression with five active chromatin marks (H3K27ac, H3K9ac, H3K4me1, H3K4me2, and DNase I hypersensitivity) across 56 biosamples, and then used these correlation links to make predictions for the predicted enhancers (regions with the "7Enh" ChromHMM state) in 127 biosamples from the Roadmap Epigenome Atlas ${ }^{38,45}$. We downloaded these predictions from www.biolchem.ucla.edu/labs/ernst/roadmaplinking and made predictions using the "confidence score".

Enhancer-gene correlation (Granja single-cell RNA and ATAC-seq). Granja et al. (2019) analyzed single-cell ATAC-seq and RNA-seq data in peripheral blood and bone marrow mononuclear cells, CD34+ bone marrow cells, and cancer cells from leukemia patients, and correlated ATAC-seq signal in distal elements with the expression of nearby genes ${ }^{39}$. We downloaded these predictions from https://github.com/GreenleafLab/MPAL-Single-Cell-2019, and used the correlation in healthy samples as the quantitative score. Cell-type specific links were not reported.

EnhancerAtlas 2.0. Gao et al. (2020) used EAGLE to predict enhancer-gene interactions across a number of human tissues and cell lines ${ }^{43}$. The method calculates a score based on six features obtained from the information of enhancers and gene expression: correlation between enhancer activity and gene expression across cell types, gene expression level of target genes, genomic distance between an enhancer and its target gene, enhancer signal, average gene activity in the region between the enhancer and target gene and enhancer-enhancer correlation. We downloaded enhancer annotations for 104 cell types from http://www.enhanceratlas.org/.

Enhancer-gene correlation (DNase-seq and microarray gene expression). Sheffield et al. (2013) correlated DNase I signal and gene expression levels using data from 112 human samples representing 72 cell types to identify regulatory elements and to predict their targets ${ }^{41}$. We 
downloaded these predictions from http://dnase.genome.duke.edu/ and used the correlation as the quantitative score. Cell-type specific links were not reported.

JEME. Cao et al. (2017) computed correlations between gene expression and various enhancer features (e.g., DNase1, H3K4me1) across multiple cell types to identify a set of putative enhancers $^{42}$. Then a sample-specific model is used to predict the enhancer gene connections in a given cell type. We downloaded the lasso-based JEME predictions in all ENCODE+Roadmap cell types from http://yiplab.cse.cuhk.edu.hk/jeme/. We used the JEME confidence score as a quantitative score.

TargetFinder. Whalen et al. 2016 built a model to predict whether nearby enhancer-promoter pairs are located at anchors of $\mathrm{Hi}-\mathrm{C}$ loops based on chromatin features ${ }^{44}$. We downloaded the TargetFinder predictions from https://raw.githubusercontent.com/shwhalen/targetfinder/master/paper/targetfinder/combined/ou tput-epw/predictions-gbm.csv. For each distal element-gene pair in our dataset, we searched to see if the element and gene TSS overlapped an enhancer and promoter loop listed in this file. If so, we assigned the pair a score corresponding to the 'prediction' column from this file; otherwise the pair received a score of 0 .

\section{Cell-type specific gene set enrichments}

We assessed whether the cell-type specificity of the ABC predictions for IBD variants could aid in identifying gene pathways enriched in IBD GWAS loci. To do so, we defined 7 cell type categories based on the biosamples available in our compendium and based on biological categories relevant IBD: mononuclear phagocytes, B cells, T cells, other hematopoietic cells, fibroblasts, epithelial cells or tissues, and other cells or tissues. We then examined the extent to which the genes predicted by $A B C$ in any cell type category, or in each individual cell type category, were enriched for gene sets from the Molecular Signatures Database ${ }^{46}$, as described above.

\section{Assessing pleiotropy across 72 traits}

We defined two classes of genes: (i) Genes linked by ABC-Max to more than one trait via a single variant. To identify such genes, we counted cases where a given variant was linked by ABC-Max to the same gene for more than one trait (that is, the linked variant has PIP $>=10 \%$ for each trait, overlaps an enhancer in a biosample that is globally enriched for that trait, and links to the same gene with the maximum $A B C$ score among genes in the locus). (ii) Genes linked to multiple traits through different variants. To identify such genes, we identified genes that were predicted by ABC-Max to be linked to at least two different traits by two different variants, where that gene was not linked to the same two traits by any single variant. (i.e., a gene linked to two traits by each of two variants would not fit this criteria). Because some of the 72 traits show high genetic correlation, we repeated these analyses in a subset of 36 traits that were selected to show pairwise genetic correlation below a threshold $\left(\left|r_{g}\right|<0.2\right)$, plus IBD. We observed similar effects in this subset of the data, where genes linked to multiple traits via different variants were more likely to have complex enhancer landscapes and large amounts of nearby noncoding genomic sequence.

\section{Assessing the effect of PPIF and e-PPIF on mitochondrial membrane potential.}

We synthesized a pool of 105 gRNAs including 40 negative control gRNAs, 9 gRNAs targeting the promoter of PPIF, and 5 gRNAs targeting the PPIF enhancer (Agilent Technologies, Inc.; see 
Table S12), cloned these gRNAs into Crop-seq-opti (Addgene \#106280), and transduced THP1 cells at a multiplicity of infection of 0.3 to ensure most cells contained $1 \mathrm{gRNA}$ integration.

For untreated and LPS-stimulated conditions, we plated $10 \mathrm{M}$ cells per replicate with $1 \mu \mathrm{g} / \mathrm{mL}$ doxycycline. After $44 \mathrm{hrs}$, we added $1 \mu \mathrm{g} / \mathrm{mL}$ LPS and harvested cells for staining $4 \mathrm{hrs}$ later. For the PMA LPS condition, we plated $10 \mathrm{M}$ cells per replicate and added $1 \mu \mathrm{g} / \mathrm{mL}$ doxycycline for 48 hrs. To differentiate into macrophage-like cells, we added fresh media with $20 \mathrm{ng} / \mathrm{mL}$ PMA and $1 \mu \mathrm{g} / \mathrm{mL}$ doxycycline for an additional $24 \mathrm{hrs}$, confirming that cells adhered to the tissue culture plate. We washed out the PMA and added fresh media with $1 \mu \mathrm{g} / \mathrm{mL}$ doxycycline and incubated cells for $45 \mathrm{hrs}$ to recover and further differentiate cells. We then added $100 \mathrm{ng} / \mathrm{mL}$ LPS for $3 \mathrm{hrs}$, harvested cells, washed $3 x$ with cold PBS, and proceeded to mitochondrial staining.

We stained cells with MitoTracker Red (200nM, Thermo Fisher, M7512) and MitoTracker Green (200nM, Thermo Fisher, M7514) according to the manufacturer's protocol and sorted cells into 3 bins according to their ratio of MitoTracker Red (which stains mitochondria dependent on $\Delta \psi_{\mathrm{m}}$ ) to MitoTracker Green (which stains mitochondria independent of $\Delta \psi_{\mathrm{m}}$ ), excluding a small population of depolarized cells with very low $\Delta \psi_{\mathrm{m}}$ (Figure S8e). We extracted genomic DNA and amplified and sequenced gRNAs from cells in each bin as previously described ${ }^{24}$.

We aligned and counted gRNAs in each bin as described above for FlowFISH experiments. For each gRNA, we summed counts across the two biological replicates. We then calculated the frequency fold-change in Fig. $\mathbf{5 d}$ and Fig. S8b by dividing gRNA reads per million by the mean value for negative-control gRNAs, and dividing values in each bin by the value for Bin 3 .

\section{Data and Code Availability}

Immune cell line ATAC-seq and H3K27ac ChIP-seq: NCBI GEO GSE155555 ABC predictions in 131 biosamples: www.engreitzlab.org/resources/

ABC code: https://github.com/broadinstitute/ABC-Enhancer-Gene-Prediction

Acknowledgements: This work was supported by the Broad Institute (E.S.L.) and an NIH Pathway to Independence Award (K99HG009917 and R00HG009917 to J.M.E.). J.M.E. was supported by the Harvard Society of Fellows and the BASE Research Initiative at the Lucile Packard Children's Hospital at Stanford University. The authors thank Larry Schweitzer, Matteo Gentili, Moshe Biton, Chris Smillie, Aviv Regev, Masahiro Kanai, Daniel Graham, Noam Shoresh, Steven Gazal, Brian Cleary, Ran Cui, Patricia Rogers, Vidya Subramanian, Gavin Schnitzler, Raj Gupta, Melina Claussnitzer, Nasa Sinnott-Armstrong, Tim Majarian, Alisa Manning, and members of the Lander Lab, Hacohen Lab, and Variant-to-Function Initiative for discussions or technical assistance. This research has been conducted using the UK Biobank Resource.

Competing interests: J.M.E., C.P.F., and E.S.L. are inventors on a patent application on CRISPR methods filed by the Broad Institute related to this work $(16 / 337,846)$. E.S.L. serves on the Board of Directors for Codiak BioSciences and Neon Therapeutics, and serves on the Scientific Advisory Board of F-Prime Capital Partners and Third Rock Ventures; he is also affiliated with several non-profit organizations including serving on the Board of Directors of the Innocence Project, Count Me In, and Biden Cancer Initiative, and the Board of Trustees for the Parker Institute for Cancer Immunotherapy. He has served and continues to serve on various federal advisory committees. C.P.F. is now an employee of Bristol Myers Squibb. 


\section{Supplemental Figures}

Average values of $A B C$ predictions within each biosample

- Values across all ABC predictions in all biosamples

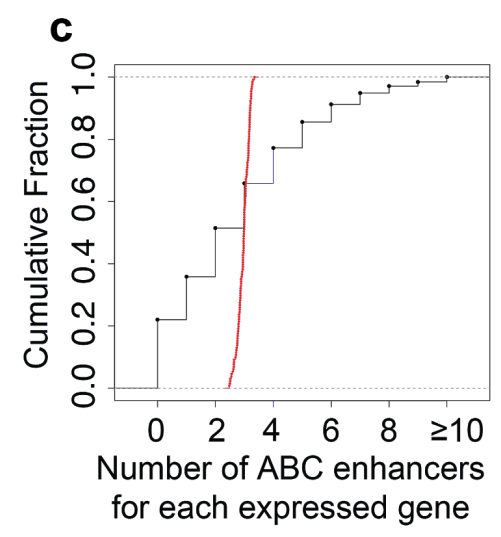

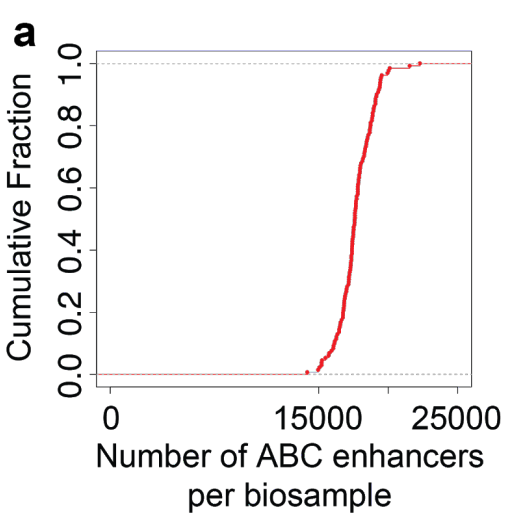
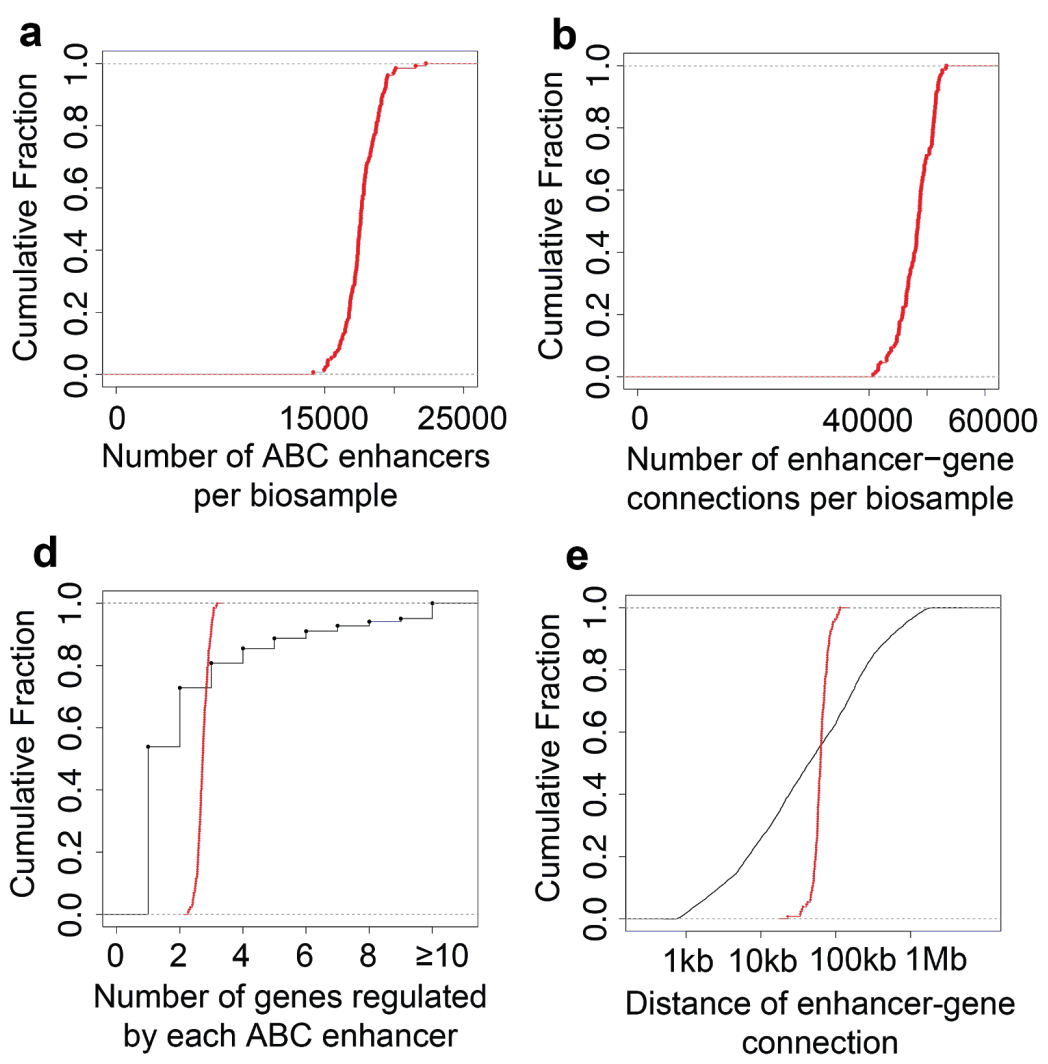

Fig S1. Properties of ABC Predictions

(a) Cumulative fraction of the number of $A B C$ enhancers within each biosample (median $=17,605$ ).

(b) Cumulative fraction of the number of enhancer-gene connections within each biosample (median = 48,441).

(c) Cumulative fractions of the number of enhancers predicted to regulate each gene across all biosamples (black line, median $=2$, mean $=2.8$ ) and the mean number of enhancers predicted to regulate each gene within each biosample (red line, median $=2.8$ ).

(d) Cumulative fractions of the number of genes regulated by each $\mathrm{ABC}$ enhancer across all genes and all biosamples (black line, median $=1$, mean $=2.7$ ) and the mean number of genes regulated by each $A B C$ enhancer within each biosample (red line, median $=2.7$ ).

(e) Cumulative fractions of the genomic distances between the enhancer and the gene for each predicted enhancer-gene connection across all genes and all biosamples (black line, median $=62,929 \mathrm{bp}$ ) and the median genomic distance between each enhancer-gene connection within each biosample (red line, median $=62,782 \mathrm{bp}$ ). 
a

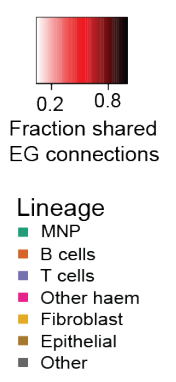

C

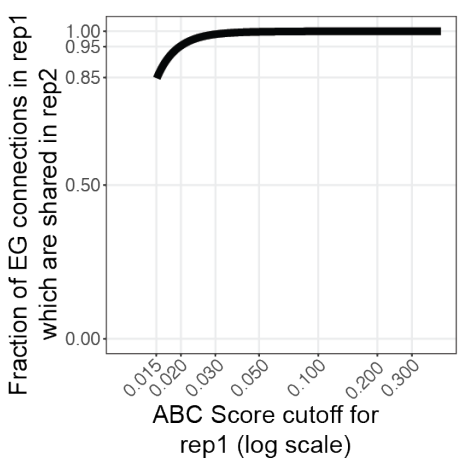

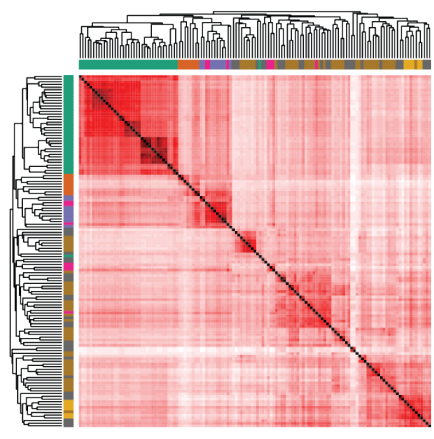

d b

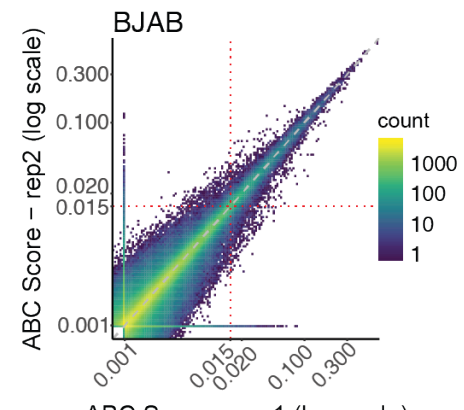

ABC Score - rep1 (log scale)

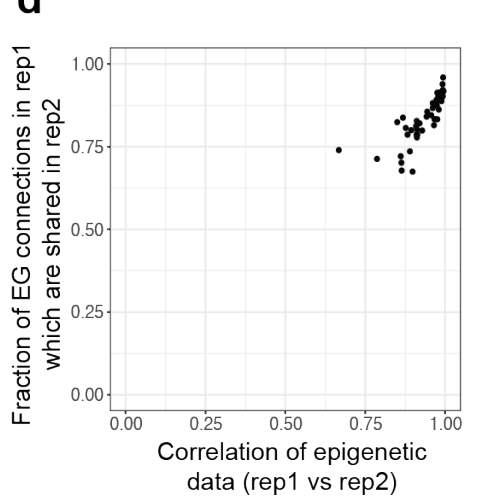

Fig S2. Distinctness and Reproducibility of ABC predictions

(a) Distinctness of predictions across biosamples. Biosample vs. Biosample $(131 \times 131)$ heatmap. The color of the $(i, j)$ pixel in the heatmap represents the fraction of enhancer-gene (EG) connections in biosample $i$ that have a corresponding overlapping prediction in biosample $j$. Two connections are considered overlapping if the predicted genes are the same and the enhancer elements overlap. Rows and columns are ordered by hierarchical clustering. A median of $19 \%$ (median of row medians) of enhancergene connections are shared across distinct biosamples.

(b) Quantitative reproducibility of $A B C$ Predictions. $A B C$ Scores computed using independent biological replicates of epigenomic data (ATAC-Seq and H3K27ac ChIP-Seq) from the BJAB cell line. Each data point is an element-gene pair.

(c) Fraction of shared enhancer-gene connections between replicates increases as ABC Score cutoff increases. X-axis: Cutoff on the ABC Score. Y-axis: For a given cutoff of the ABC Score, the fraction of element-gene pairs with an $A B C$ score greater than the cutoff in replicate 1 that have an $A B C$ score > 0.015 in replicate 2 . Separate curves are computed for each biosample and then the average across biosamples is plotted.

(d) Fraction of shared enhancer-gene connections increases as reproducibility of underlying epigenetic data increases. Each data point represents a biosample. X-axis: average correlation of ATACSeq (or DNase-Seq) and H3K27ac ChIP-Seq signal in candidate regions computed using replicate epigenetic experiments. Y-axis: Fraction of E-G connections with ABC Score $>0.015$ in replicate 1 which also have $A B C$ Score $>0.015$ in replicate 2. 

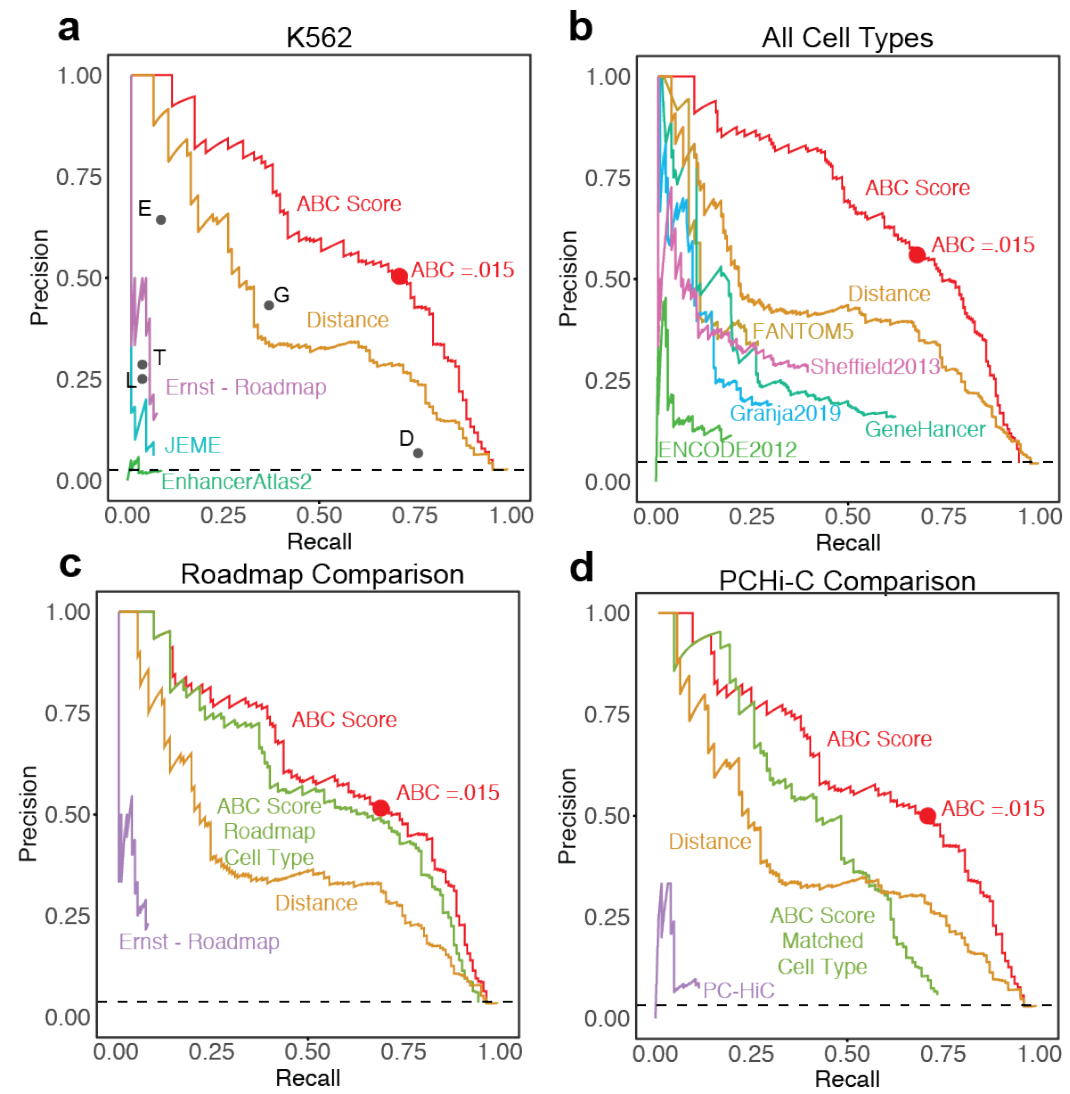

Fig. S3. ABC performs well at identifying regulatory enhancer-gene connections in CRISPR datasets.

(a) Comparison of enhancer-gene predictors to experimental CRISPR data in K562 cells. Each of these predictors makes K562-specific predictions. Curves represent continuous predictors. Dots represent binary predictors as follows: $(E)$ Each gene is predicted to be regulated only by the element closest to its transcription start site, $(G)$ each element is predicted to regulate only the nearest (to TSS) expressed gene, (T) TargetFinder method ${ }^{44}$, (L) elements and genes at opposite ends of HiCCUPS loops are predicted as a connection $^{89}$, (D) each element is predicted to regulate each gene in the same contact domains ${ }^{89}$. Red dot on $\mathrm{ABC}$ score curve represents the precision and recall achieved using a threshold on the $\mathrm{ABC}$ score of 0.015 .

(b) Comparison of $A B C$ and other enhancer-gene predictors in full CRISPR dataset. Comparison of enhancer-gene predictors to experimental CRISPR data in K562, GM12878, NCCIT, BJAB (+/- stimulation), Jurkat (+/- stimulation), THP1 (+/- stimulation) cells and primary hepatocytes. For ABC, we used the predictions in the cell type corresponding to the CRISPR experiments. Because ABC is the only method that makes predictions in all of these cell types, we used this plot to compare $A B C$ to other methods that make predictions without cell-type information. We consider each enhancer-gene pair predicted by these methods to be a prediction in all cell types. Red dot on ABC score curve as in (a).

(c) Comparison of $\mathrm{ABC}$ and Ernst-Roadmap predictions. Comparison of enhancer-gene predictors to experimental CRISPR data in K562, GM12878, and unstimulated Jurkat, BJAB, THP1 cells. Red line represents comparison of $A B C$ scores derived using epigenetic data in the same cell type as the CRISPR experiment was performed. Red dot on $A B C$ score curve as in (a). To compare Roadmap predictions to CRISPR data, we made cell type substitutions because the Roadmap predictions did not include BJAB, Jurkat, and THP1 cells: for BJAB CRISPR data we compared to predictions in the Roadmap B cell sample (E032); for THP1 data we used the Roadmap monocyte sample (E124); and for Jurkat data we used the Roadmap T cell sample (E034). To directly compare the performance of ABC and Ernst-Roadmap methods in matched cell types, we also calculated $A B C$ performance using the same cell type substitutions (green line). 
bioRxiv preprint doi: https://doi.org/10.1101/2020.09.01.278093; this version posted September 3, 2020. The copyright holder for this preprint (which was not certified by peer review) is the author/funder, who has granted bioRxiv a license to display the preprint in perpetuity. It is made available under aCC-BY-ND 4.0 International license.

(d) Comparison of $\mathrm{ABC}$ to Promoter-Capture Hi-C. Comparison of enhancer-gene predictors to experimental CRISPR data in K562 and unstimulated BJAB, THP1 and Jurkat cells. Red line represents comparison of $A B C$ Scores derived using epigenetic data in the same cell type as the CRISPR experiment was performed. Red dot on ABC Score curve as in (a). To compare promoter-capture Hi-C predictions to CRISPR data, we made cell type substitutions because the these predictions did not include K562, BJAB, Jurkat, and THP1 cells: for K562 CRISPR data we compared to predictions in erythroblasts; for BJAB CRISPR data we compared to total B cells; for THP1 data we compared to monocytes; and for Jurkat data we compared to total CD4+ T cells. To directly compare the performance of $A B C$ and PC-HiC methods in matched cell types, we also calculated $A B C$ performance using the same cell type substitutions (green line). 
a

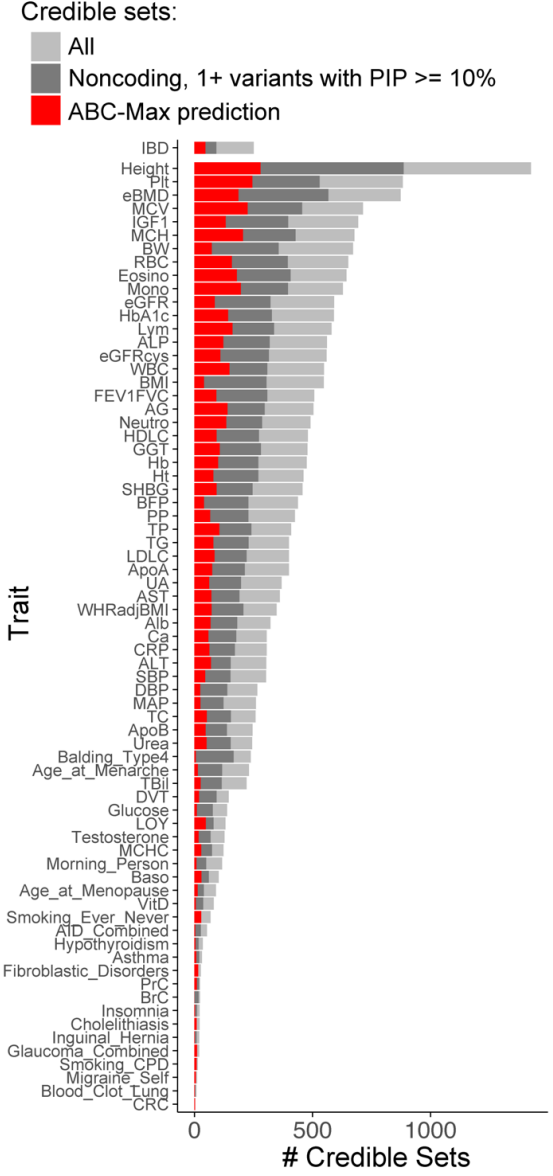

b

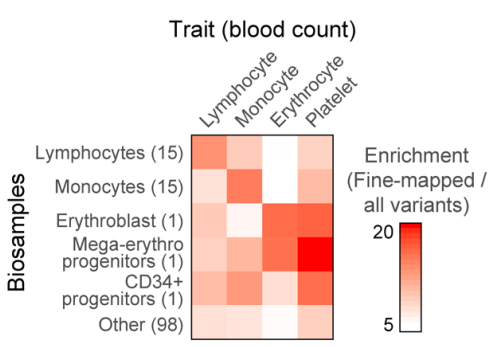

C

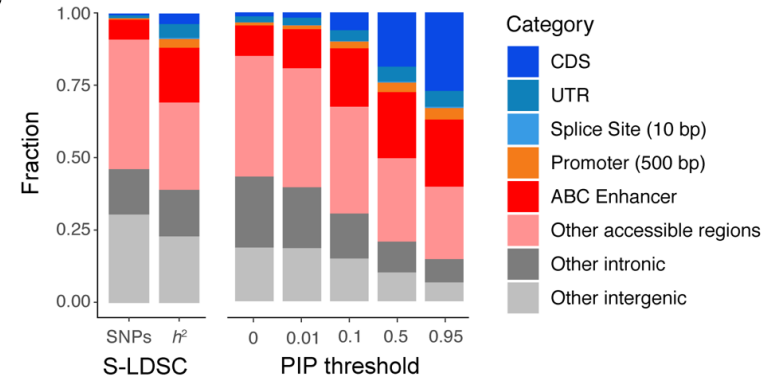

d

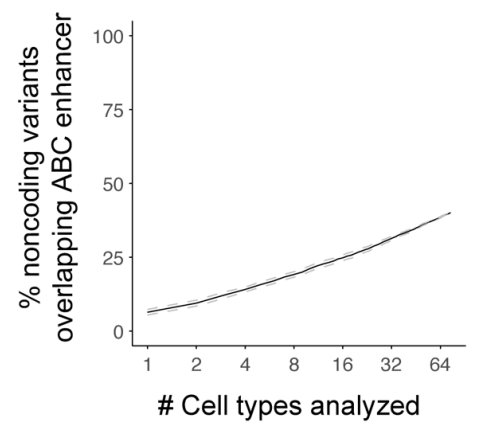

Figure S4. Fine-mapped GWAS variants are highly enriched in ABC enhancers.

(a) Number of credible sets analyzed for 72 diseases and complex traits. Light gray shows total number of fine-mapped credible sets. Dark gray shows number of such credible sets with no coding or splice site variants, and at least one variant with PIP $>=10 \%$. Red shows number of credible sets for which ABC-Max makes a prediction (i.e., a variant with PIP $>=10 \%$ overlaps an $\mathrm{ABC}$ enhancer in a biosample that shows global enrichment for that trait). See Table $\mathbf{S 7}$ for trait descriptions and additional statistics.

(b) Enrichment of fine-mapped variants (PIP >= 10\%) associated with 4 blood cell traits in $A B C$ enhancers in the corresponding blood cell types or progenitors. Enrichment $=$ (fraction of fine-mapped variants / fraction of all common variants) overlapping regions in each cell type. Numbers of biosamples in each category are shown in parentheses.

(c) Fraction of variants or heritability for all 72 traits contained in different categories of genomic regions: coding sequences (CDS), untranslated regions (UTR), splice sites (within $10 \mathrm{bp}$ of a intron-exon junction of a protein-coding gene), promoters ( $\pm 250 \mathrm{bp}$ from the gene TSS), ABC enhancers in 131 biosamples, other accessible regions not called as $A B C$ enhancers, and other intronic or intergenic regions. In cases where a variant overlaps more than one category, the variant was assigned to the first category that it overlapped (i.e., variants in coding sequences were not also counted as overlapping ABC enhancers, see Methods). Left: All common variants (1000 Genomes) or heritability $\left(h^{2}\right.$, as estimated by S-LDSC in inversevariance weighted meta-analysis across 74 traits). Right: Fraction of variants above a threshold on the finemapping PIP.

(d) \% of noncoding variants across all traits that overlap an $A B C$ enhancer in an enriched biosample, as a function of the number of cell types analyzed. Biosamples (131) were grouped into 74 cell types/tissues; and analyzed in random order. Black line: mean across 20 random orderings. Dashed gray lines: $95 \%$ confidence intervals. 
bioRxiv preprint doi: https://doi.org/10.1101/2020.09.01.278093; this version posted September 3, 2020. The copyright holder for this preprint (which was not certified by peer review) is the author/funder, who has granted bioRxiv a license to display the preprint in perpetuity. It is made available under aCC-BY-ND 4.0 International license.

a

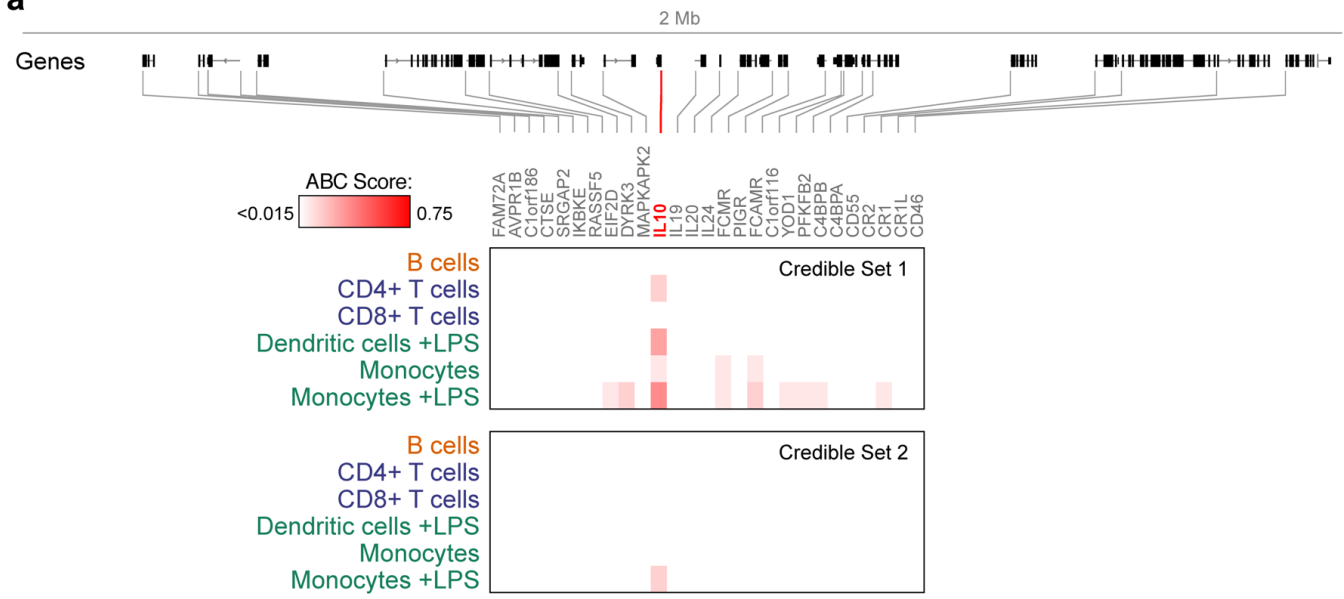

b

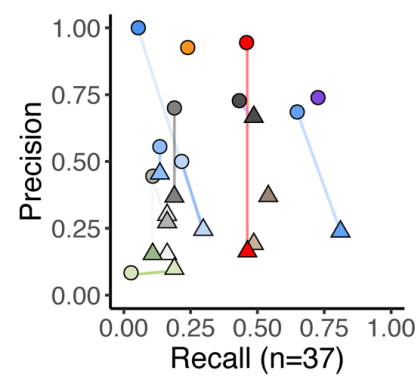

Method:
ABC
ABC (no immune, no gut)
Closest TSS
eQTL:
0 JLIM (Chun 2017)
coloc (OpenTargets)
HEIDI (Hauberg 2017)
multiXcan (Barbeira 2019)
$3 D$ loops:
0 COGS (Javierre 2016)
Promoter-capture Hi-C (Javierre 2016)

Best score per locus

$\triangle$ Global threshold

E-G prediction - Correlation:

O Roadmap ChromHMM-RNA (Liu 2017)

ScATAC-scRNA (Granja 2019)

O DNase-RNA (Sheffield 2013)

O FANTOM eRNA (Andersson 2014)

O ENCODE DNase (Thurman 2012)

E-G prediction - Other:

JEME (Cao 2017)

Enhancer Atlas (Gao 2020)

Figure S5. ABC enhancer maps connect GWAS variants to IBD genes.

(a) ABC predictions for IBD credible sets linked to IL10. Heatmap shows ABC scores for each gene within $1 \mathrm{Mb}$ in selected primary immune cell types. Credible Set 1 is linked by ABC to multiple genes, but IL10 (red) has the strongest ABC score in any cell type.

(b) Precision-recall plot for identifying known IBD genes, comparing additional variations on the prediction methods (superset of data in Fig. 2a). For ABC, we compared ABC-Max (assigning each credible set to the gene with the maximum $A B C$ score, red circle), $A B C-M a x$ excluding all immune and gut tissue biosamples (orange circle), and ABC-All (assigning each credible set to all genes linked to enhancers, red triangle). For other methods that provided quantitative scores, we similarly compared choosing the gene with the best score per locus (circles) with choosing all genes above the global thresholds previously reported in each study (triangles). In most cases, the best gene per locus outperformed using a global threshold. 
a

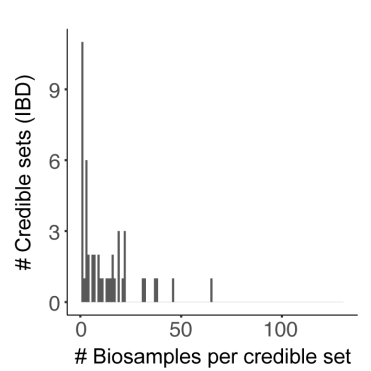

C

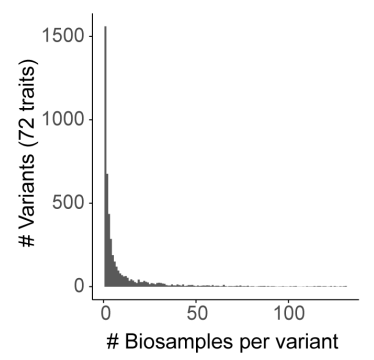

b

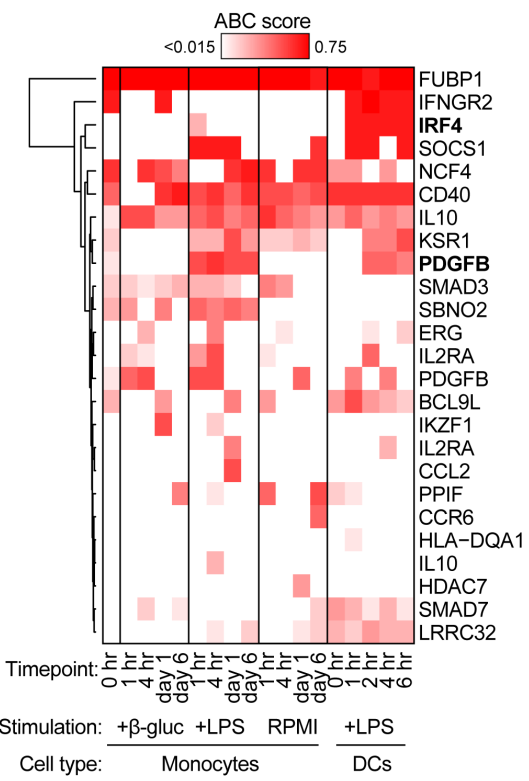

d $370 \mathrm{~Kb}$

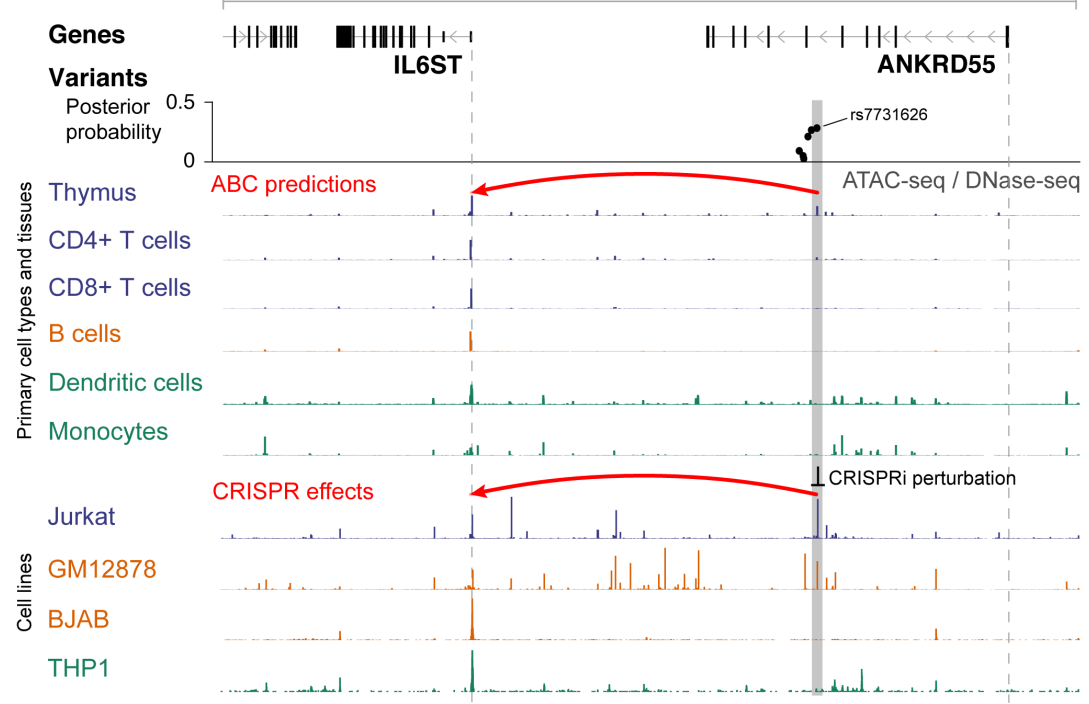

Figure S6. Cell-type specificity of $A B C$ predictions.

(a) Histogram of number of biosamples predicted by ABC-Max per noncoding IBD credible set (total $=47$ credible sets).

(b) Heatmap of $A B C$ scores for predicted IBD genes in resting and stimulated mononuclear phagocytes (from epigenomic data in monocytes ${ }^{90}$ and dendritic cells ${ }^{91}$ ). IRF4 and PDGFB (bold) are two examples where $A B C$ predictions are specific to a particular stimulated state (+LPS) and are not observed in unstimulated states.

(c) Histogram of number of biosamples predicted by $A B C$-Max per variant (total $=4,976$ unique variants across 72 traits).

(d) A variant in an intron of $A N K R D 55$ is predicted by the ABC Model to regulate IL6ST in thymus. Gray bar highlights the variant overlapping the predicted $A B C$ enhancer. Vertical dotted lines represent TSSs. Red arc at top denotes ABC-Max prediction. Rec arc at bottom denotes that CRISPRi of the highlighted enhancer significantly affects the expression of IL6ST only in Jurkat cells. 
bioRxiv preprint doi: https://doi.org/10.1101/2020.09.01.278093; this version posted September 3, 2020. The copyright holder for this preprint (which was not certified by peer review) is the author/funder, who has granted bioRxiv a license to display the preprint in perpetuity. It is made available under aCC-BY-ND 4.0 International license.

a

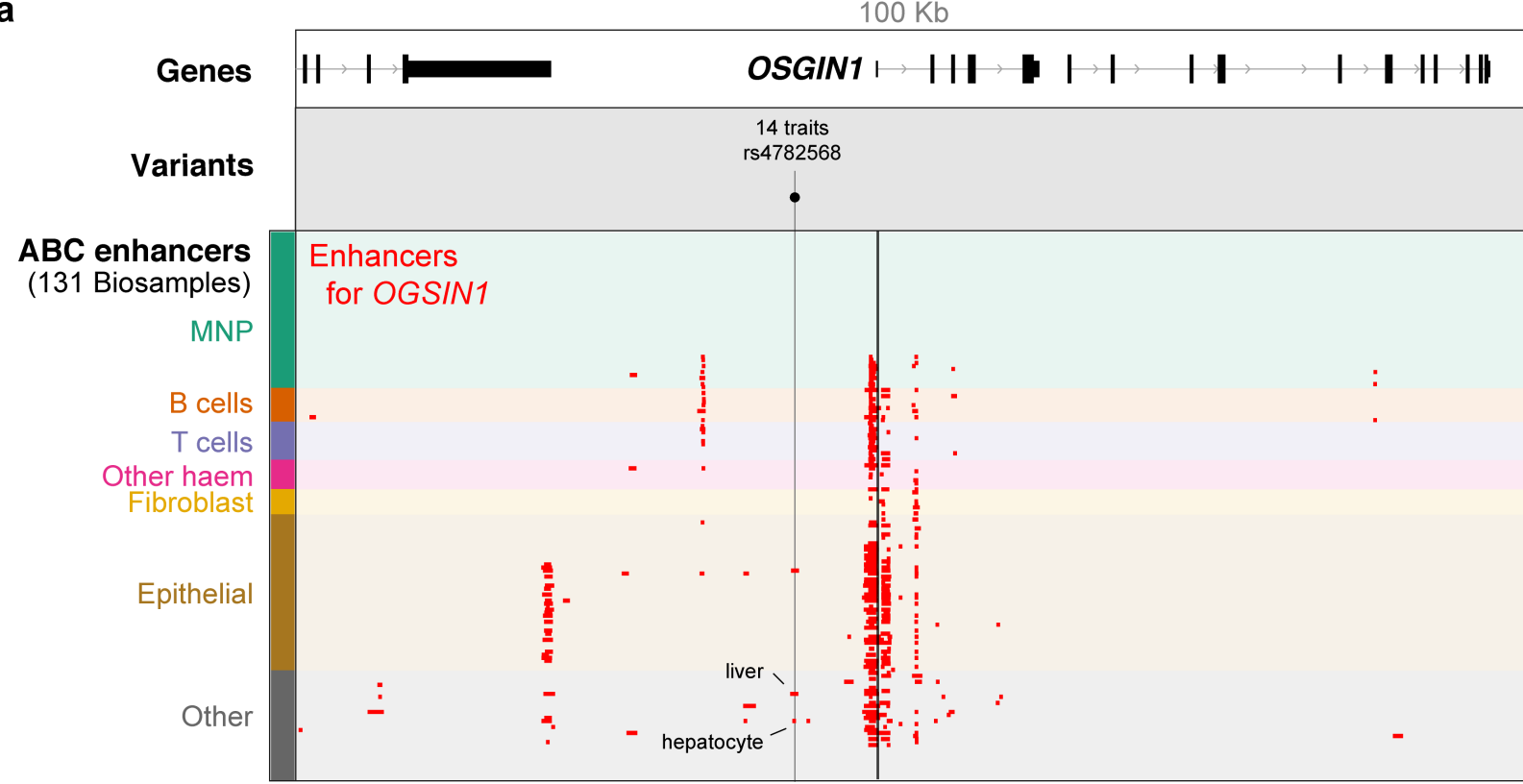

b

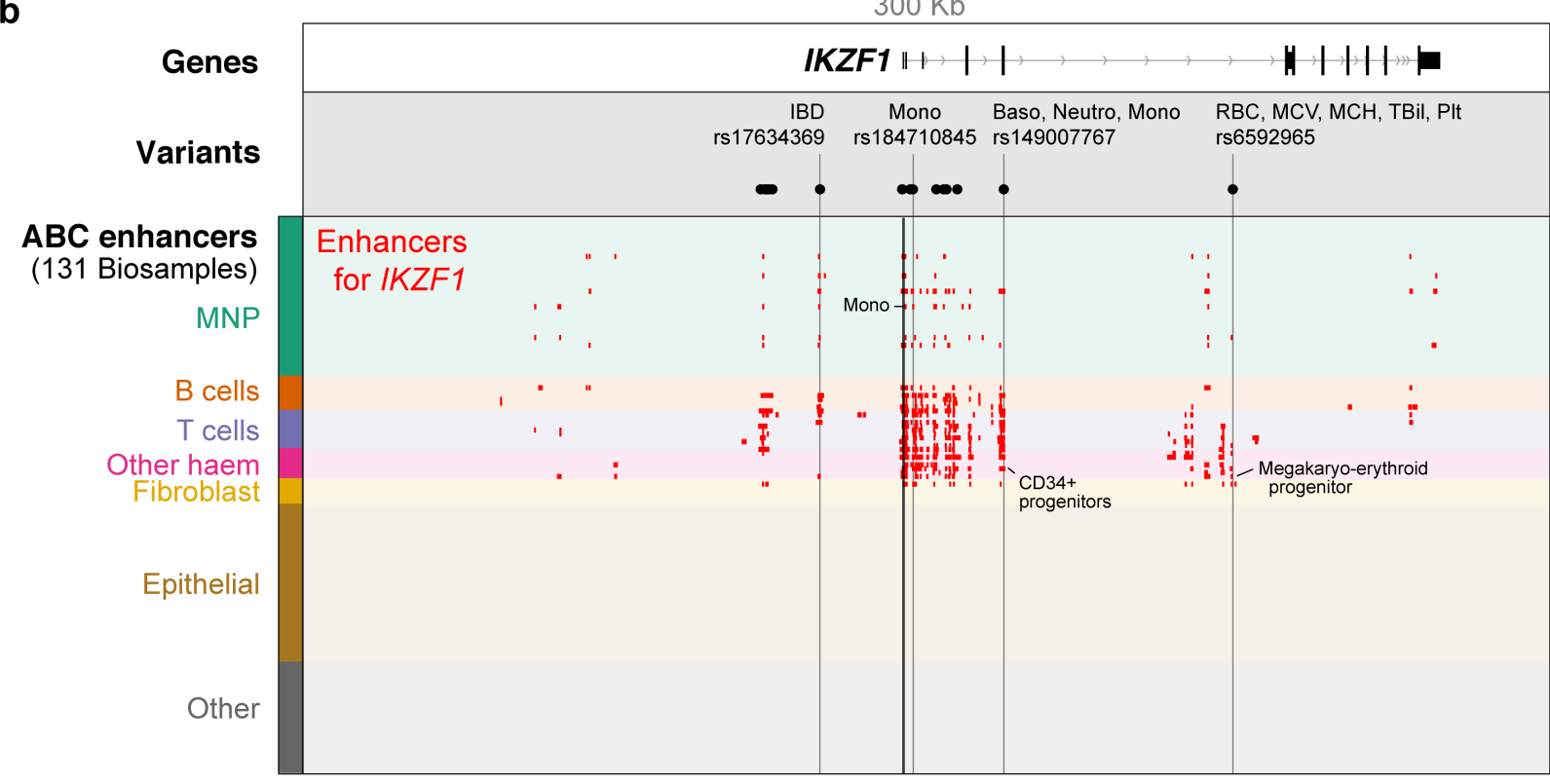

C

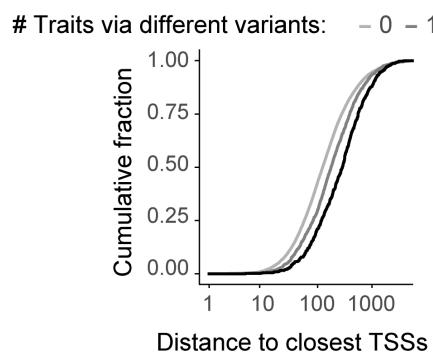

$(\mathrm{Kb})$ d

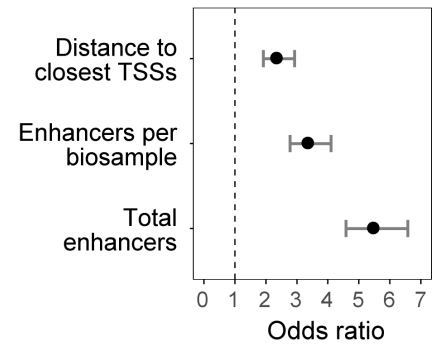


Figure S7. Genes linked by ABC to multiple traits.

(a) ABC links OSGIN1 to 14 traits via rs4782568. Red boxes mark enhancers predicted to regulate OSGIN1. Thick black line marks the OSGIN1 TSS.

(b) Same for IKZF1 locus. Black dots mark fine-mapped noncoding variants (PIP >= 10\%) associated with one or more traits linked to IKZF1 by ABC-Max.

(c) Distance between the TSSs of neighboring genes for each gene linked by ABC-Max to zero traits, one trait, or two or more traits through different variants.

(d) The complexity of a gene's enhancer landscape is correlated with the odds of the gene being linked to multiple GWAS traits. Wald odds ratios and $95 \% \mathrm{Cls}$ for the genes in the top decile, compared to other genes, are shown for 3 gene-based metrics: the total number of enhancers linked to the gene by ABC in any biosample, the number of enhancers linked to a gene per biosample in which the gene's promoter is active, and genomic distance to the nearest neighboring TSS on either side of the gene. 
bioRxiv preprint doi: https://doi.org/10.1101/2020.09.01.278093; this version posted September 3, 2020. The copyright holder for this preprint (which was not certified by peer review) is the author/funder, who has granted bioRxiv a license to display the preprint in perpetuity. It is made available under aCC-BY-ND 4.0 International license.

a

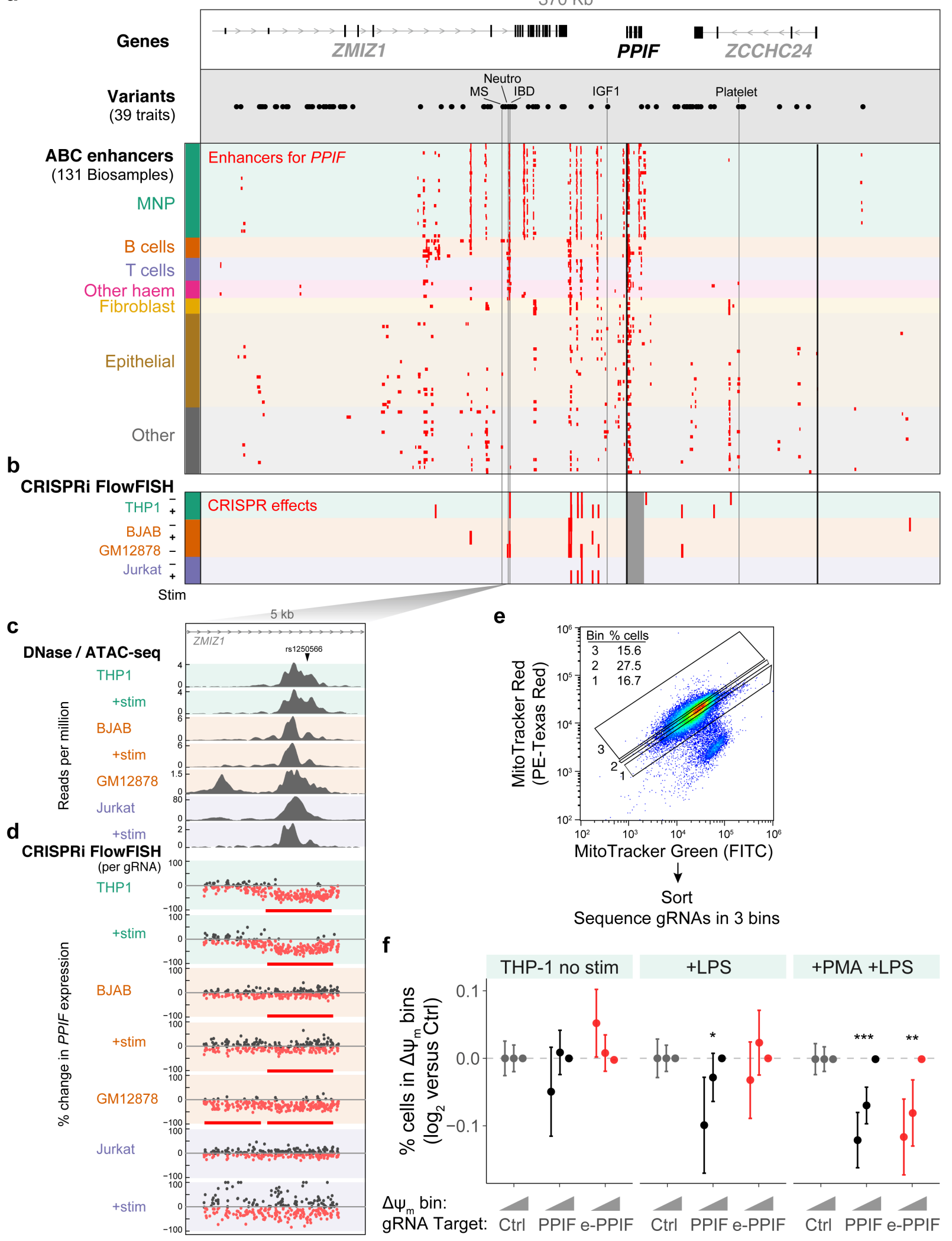


Figure S8. Enhancers and variants connected to PPIF.

(a) ABC predictions for variants near PPIF. Black dots represent either (i) fine-mapped variants (PIP >= $10 \%)$ for IBD and UK Biobank traits, or (ii) lead variants for any phenotype from the GWAS Catalog ${ }^{92}$. "IBD" label points to rs1250566. Red boxes mark enhancers predicted to regulate PPIF. Thick back lines mark TSSs. Thin black lines mark selected variants.

(b) CRISPRi-FlowFISH data for PPIF in 7 immune cell lines and stimulated states. Red boxes mark distal enhancers (CRISPR gRNAs lead to a significant decrease in the expression of PPIF). Dark gray box marks the gene body of PPIF, where CRISPRi cannot accurately assess the effects of putative regulatory elements ${ }^{24}$.

(c) Chromatin accessibility in 5-kb regions around the PPIF enhancer and promoter. Signal tracks show ATAC-seq (for THP1 and BJAB) or DNase-seq (for GM12878 and Jurkat) data in reads per million.

(d) Effect of each tested gRNA on PPIF expression, as measured by CRISPRi-FlowFISH (see Methods). Dots: gRNAs whose effect estimate is $>0 \%$ (black) or $<0 \%$ (red). Red bars show regions where gRNAs have a significant effect on gene expression (FDR $<0.05$ ), as compared by a two-sided t-test to negative control gRNAs.

(e) Schema of pooled CRISPRi screen to examine the effects of PPIF and e-PPIF on mitochondrial membrane potential $\left(\Delta \psi_{\mathrm{m}}\right)$. Cells expressing a pool of gRNAs were stained with MitoTracker Red and MitoTracker Green and sorted into 3 bins of increasing Red:Green ratios. gRNAs from cells in each bin were PCR-amplified, sequenced, and counted.

(f) Effects of CRISPRi gRNAs (targeting e-PPIF, PPIF promoter, or negative controls (Ctrl)) on $\Delta \psi_{\mathrm{m}}$, quantified as the frequency of THP1 cells carrying those gRNAs with low or medium versus high MitoTracker Red signal (corresponding to Bins 1, 2, and 3, respectively; superset of data in Fig. 5d). We tested THP1 cells in unstimulated conditions, stimulated with LPS, and differentiated with PMA and stimulated with LPS (see Methods). Error bars: 95\% confidence intervals for the mean of 40, 9, and 5 gRNAs for Ctrl, PPIF, and e-PPIF, respectively. Two-sided rank-sum $P<0.05\left(^{*}\right),<0.01\left(^{(*}\right)$, or $<0.005\left(^{(*}\right)$ versus Ctrl. 


\section{Supplementary Tables}

Table S1 | Epigenomic data collected in immune cell lines.

Table S2 | Metrics for ABC predictions in 131 biosamples.

Table S3 | CRISPRi-FlowFISH data: data per guide.

Table S4 | CRISPRi-FlowFISH data: summary per candidate element.

Table S5 | Comparison of CRISPR data to enhancer-gene predictions.

Table S6 | Enrichment of GWAS variants in ABC enhancers across biosamples.

Table S7 | Summary of diseases and traits.

Table S8 | ABC predictions for IBD GWAS loci.

Table S9 | ABC-Max predictions for 72 diseases and complex traits.

Table S10 | References linking predicted genes to effects on experimental colitis.

Table S11 | ABC and ABC-Max metrics for all genes.

Table S12 | PPIF and mitochondrial membrane potential: CRISPRi data per guide. 


\section{References}

1 Claussnitzer, M. et al. A brief history of human disease genetics. Nature 577, 179-189, doi:10.1038/s41586-019-1879-7 (2020).

2 Duerr, R. H. et al. A Genome-Wide Association Study Identifies IL23R as an Inflammatory Bowel Disease Gene. Science 314, 1461-1463, doi:10.1126/science.1135245 (2006).

3 Mohanan, V. et al. C1orf106 is a colitis risk gene that regulates stability of epithelial adherens junctions. Science 359, 1161-1166, doi:10.1126/science.aan0814 (2018).

4 Cadwell, K. et al. A key role for autophagy and the autophagy gene Atg16/1 in mouse and human intestinal Paneth cells. Nature 456, 259-263, doi:10.1038/nature07416 (2008).

5 Kuballa, P., Huett, A., Rioux, J. D., Daly, M. J. \& Xavier, R. J. Impaired autophagy of an intracellular pathogen induced by a Crohn's disease associated ATG16L1 variant. PLoS One 3, e3391, doi:10.1371/journal.pone.0003391 (2008).

6 Saitoh, T. et al. Loss of the autophagy protein Atg16L1 enhances endotoxin-induced IL-1beta production. Nature 456, 264-268, doi:10.1038/nature07383 (2008).

7 Hampe, J. et al. A genome-wide association scan of nonsynonymous SNPs identifies a susceptibility variant for Crohn disease in ATG16L1. Nat Genet 39, 207-211, doi:10.1038/ng1954 (2007).

8 Graham, D. B. \& Xavier, R. J. Pathway paradigms revealed from the genetics of inflammatory bowel disease. Nature 578, 527-539, doi:10.1038/s41586-020-2025-2 (2020).

9 Musunuru, K. et al. From noncoding variant to phenotype via SORT1 at the $1 \mathrm{p} 13$ cholesterol locus. Nature 466, 714-719, doi:10.1038/nature09266 (2010).

10 Claussnitzer, M. et al. FTO Obesity Variant Circuitry and Adipocyte Browning in Humans. The New England journal of medicine 373, 895-907, doi:10.1056/NEJMoa1502214 (2015).

11 Gupta, R. M. et al. A Genetic Variant Associated with Five Vascular Diseases Is a Distal Regulator of Endothelin-1 Gene Expression. Cell 170, 522-533.e515, doi:10.1016/j.cell.2017.06.049 (2017).

12 Sekar, A. et al. Schizophrenia risk from complex variation of complement component 4. Nature 530, 177-183, doi:10.1038/nature16549 (2016).

13 Farh, K. K.-H. et al. Genetic and epigenetic fine mapping of causal autoimmune disease variants. Nature 518, 337-343, doi:10.1038/nature13835 (2015).

14 Maurano, M. T. et al. Systematic localization of common disease-associated variation in regulatory DNA. Science (New York, N.Y.) 337, 1190-1195, doi:10.1126/science.1222794 (2012).

15 Consortium, E. P. An integrated encyclopedia of DNA elements in the human genome. Nature 489, 57-74, doi:10.1038/nature11247 (2012).

16 Thurman, R. E. et al. The accessible chromatin landscape of the human genome. Nature 489, 7582, doi:10.1038/nature11232 (2012).

17 Gasperini, M., Tome, J. M. \& Shendure, J. Towards a comprehensive catalogue of validated and target-linked human enhancers. Nat Rev Genet, doi:10.1038/s41576-019-0209-0 (2020).

18 Moore, J. E., Pratt, H. E., Purcaro, M. J. \& Weng, Z. A curated benchmark of enhancer-gene interactions for evaluating enhancer-target gene prediction methods. Genome Biol 21, 17, doi:10.1186/s13059-019-1924-8 (2020).

19 Westra, H.-J. \& Franke, L. From genome to function by studying eQTLs. Biochimica et Biophysica Acta (BBA) - Molecular Basis of Disease 1842, 1896-1902, doi:10.1016/j.bbadis.2014.04.024 (2014).

20 Schaid, D. J., Chen, W. \& Larson, N. B. From genome-wide associations to candidate causal variants by statistical fine-mapping. Nat Rev Genet 19, 491-504, doi:10.1038/s41576-018-0016-z (2018). 
Huang, $\mathrm{H}$. et al. Fine-mapping inflammatory bowel disease loci to single-variant resolution. Nature 547, 173-178, doi:10.1038/nature22969 (2017).

22 Consortium, W. T. C. C. et al. Bayesian refinement of association signals for 14 loci in 3 common diseases. Nature genetics 44, 1294-1301, doi:10.1038/ng.2435 (2012).

23 Ulirsch, J. C. et al. Interrogation of human hematopoiesis at single-cell and single-variant resolution. Nat Genet 51, 683-693, doi:10.1038/s41588-019-0362-6 (2019).

24 Fulco, C. P. et al. Activity-by-contact model of enhancer-promoter regulation from thousands of CRISPR perturbations. Nat Genet 51, 1664-1669, doi:10.1038/s41588-019-0538-0 (2019).

Rescigno, M. \& Di Sabatino, A. Dendritic cells in intestinal homeostasis and disease. J Clin Invest 119, 2441-2450, doi:10.1172/JCl39134 (2009).

Steinbach, E. C. \& Plevy, S. E. The role of macrophages and dendritic cells in the initiation of inflammation in IBD. Inflamm Bowel Dis 20, 166-175, doi:10.1097/MIB.0b013e3182a69dca (2014).

Smillie, C. S. et al. Intra- and Inter-cellular Rewiring of the Human Colon during Ulcerative Colitis. Cell 178, 714-730 e722, doi:10.1016/j.cell.2019.06.029 (2019).

Ulirsch, J. C. et al. Systematic Functional Dissection of Common Genetic Variation Affecting Red Blood Cell Traits. Cell 165, 1530-1545, doi:10.1016/j.cell.2016.04.048 (2016).

Zhou, J. et al. Deep learning sequence-based ab initio prediction of variant effects on expression and disease risk. Nat Genet 50, 1171-1179, doi:10.1038/s41588-018-0160-6 (2018).

Kelley, D. R., Snoek, J. \& Rinn, J. L. Basset: learning the regulatory code of the accessible genome with deep convolutional neural networks. Genome research 26, 990-999, doi:10.1101/gr.200535.115 (2016).

31 Wainberg, M. et al. Opportunities and challenges for transcriptome-wide association studies. Nat Genet 51, 592-599, doi:10.1038/s41588-019-0385-z (2019).

Barbeira, A. N. et al. Integrating predicted transcriptome from multiple tissues improves association detection. PLoS Genet 15, e1007889, doi:10.1371/journal.pgen.1007889 (2019).

O'Connor, L. J. et al. Extreme Polygenicity of Complex Traits Is Explained by Negative Selection. Am J Hum Genet 105, 456-476, doi:10.1016/j.ajhg.2019.07.003 (2019).

Stacey, D. et al. ProGeM: a framework for the prioritization of candidate causal genes at molecular quantitative trait loci. Nucleic Acids Res 47, e3, doi:10.1093/nar/gky837 (2019).

Chun, S. et al. Limited statistical evidence for shared genetic effects of eQTLs and autoimmunedisease-associated loci in three major immune-cell types. Nature genetics 49, 600-605, doi:10.1038/ng.3795 (2017).

36 Carvalho-Silva, D. et al. Open Targets Platform: new developments and updates two years on. Nucleic Acids Research 47, D1056-D1065, doi:10.1093/nar/gky1133 (2019).

37 Hauberg, M. E. et al. Large-Scale Identification of Common Trait and Disease Variants Affecting Gene Expression. Am J Hum Genet 101, 157, doi:10.1016/j.ajhg.2017.06.003 (2017). in human regulatory domains. Genome Biol 18, 193, doi:10.1186/s13059-017-1308-x (2017).

Granja, J. M. et al. Single-cell multiomic analysis identifies regulatory programs in mixedphenotype acute leukemia. Nat Biotechnol 37, 1458-1465, doi:10.1038/s41587-019-0332-7 (2019).

40 Andersson, R. et al. An atlas of active enhancers across human cell types and tissues. Nature 507, 455-461, doi:10.1038/nature12787 (2014).

41 Sheffield, N. C. et al. Patterns of regulatory activity across diverse human cell types predict tissue identity, transcription factor binding, and long-range interactions. Genome research 23, 777-788, doi:10.1101/gr.152140.112 (2013).

42 Cao, Q. et al. Reconstruction of enhancer-target networks in 935 samples of human primary cells, tissues and cell lines. Nat Genet 49, 1428-1436, doi:10.1038/ng.3950 (2017). 
Gao, T. \& Qian, J. EnhancerAtlas 2.0: an updated resource with enhancer annotation in 586 tissue/cell types across nine species. Nucleic Acids Res 48, D58-D64, doi:10.1093/nar/gkz980 (2020).

44 Whalen, S., Truty, R. M. \& Pollard, K. S. Enhancer-promoter interactions are encoded by complex genomic signatures on looping chromatin. Nature genetics, doi:10.1038/ng.3539 (2016).

Roadmap Epigenomics, C. et al. Integrative analysis of 111 reference human epigenomes. Nature 518, 317-330, doi:10.1038/nature14248 (2015). doi:10.1093/bioinformatics/btr260 (2011).

Consortium, G. et al. Genetic effects on gene expression across human tissues. Nature 550, 204213, doi:10.1038/nature24277 (2017).

Engreitz, J. M. et al. Local regulation of gene expression by IncRNA promoters, transcription and splicing. Nature, doi:10.1038/nature20149 (2016).

de Lange, K. M. et al. Genome-wide association study implicates immune activation of multiple integrin genes in inflammatory bowel disease. Nature genetics 49, 256-261, doi:10.1038/ng.3760 (2017). Cells. Cell 167, 1398-1414.e1324, doi:10.1016/j.cell.2016.10.026 (2016).

Greene, C. S. et al. Understanding multicellular function and disease with human tissue-specific networks. Nat Genet 47, 569-576, doi:10.1038/ng.3259 (2015). doi:10.1038/ng.2355 (2012).

53 Watanabe, K. et al. A global overview of pleiotropy and genetic architecture in complex traits. Nat Genet 51, 1339-1348, doi:10.1038/s41588-019-0481-0 (2019).

Solovieff, N., Cotsapas, C., Lee, P. H., Purcell, S. M. \& Smoller, J. W. Pleiotropy in complex traits: challenges and strategies. Nature Reviews Genetics 14, 483-495, doi:10.1038/nrg3461 (2013). genetics. Nature Reviews Drug Discovery 12, 581-594, doi:10.1038/nrd4051 (2013).

Brennan, M. S., Matos, M. F., Richter, K. E., Li, B. \& Scannevin, R. H. The NRF2 transcriptional target, OSGIN1, contributes to monomethyl fumarate-mediated cytoprotection in human astrocytes. Sci Rep 7, 42054, doi:10.1038/srep42054 (2017). interference. Science 354, 769-773, doi:10.1126/science.aag2445 (2016).

Wang, X. \& Goldstein, D. B. Enhancer Domains Predict Gene Pathogenicity and Inform Gene Discovery in Complex Disease. Am J Hum Genet 106, 215-233, doi:10.1016/j.ajhg.2020.01.012 (2020).

Elrod, J. W. \& Molkentin, J. D. Physiologic functions of cyclophilin D and the mitochondrial permeability transition pore. Circ J 77, 1111-1122, doi:10.1253/circj.cj-13-0321 (2013).

60 Baines, C. P. et al. Loss of cyclophilin D reveals a critical role for mitochondrial permeability transition in cell death. Nature 434, 658-662, doi:10.1038/nature03434 (2005).

61 Nakagawa, T. et al. Cyclophilin D-dependent mitochondrial permeability transition regulates some necrotic but not apoptotic cell death. Nature 434, 652-658, doi:10.1038/nature03317 (2005).

62 Zhu, X., Hogan, S. P., Molkentin, J. D. \& Zimmermann, N. Cyclophilin D regulates necrosis, but not apoptosis, of murine eosinophils. American journal of physiology. Gastrointestinal and liver physiology 310, G609-617, doi:10.1152/ajpgi.00389.2015 (2016).

63 Briston, T., Selwood, D. L., Szabadkai, G. \& Duchen, M. R. Mitochondrial Permeability Transition: A Molecular Lesion with Multiple Drug Targets. Trends Pharmacol Sci 40, 50-70, doi:10.1016/j.tips.2018.11.004 (2019). 
Sandborn, W. J. \& Tremaine, W. J. Cyclosporine Treatment of Inflammatory Bowel Disease. Mayo Clinic Proceedings 67, 981-990, doi:10.1016/s0025-6196(12)60930-6 (1992).

65 Schreiber, S. L. \& Crabtree, G. R. The mechanism of action of cyclosporin A and FK506. Immunol Today 13, 136-142, doi:10.1016/0167-5699(92)90111-J (1992).

66 Chen, $T$. et al. Cyclosporin A impairs dendritic cell migration by regulating chemokine receptor expression and inhibiting cyclooxygenase-2 expression. Blood 103, 413-421, doi:10.1182/blood2003-07-2412 (2004).

67 Duperrier, K. et al. Cyclosporin A inhibits dendritic cell maturation promoted by TNF-alpha or LPS but not by double-stranded RNA or CD40L. J Leukoc Biol 72, 953-961 (2002).

68 Siepel, A. et al. Evolutionarily conserved elements in vertebrate, insect, worm, and yeast genomes. Genome Res 15, 1034-1050, doi:10.1101/gr.3715005 (2005).

69 Dimas, A. S. et al. Common regulatory variation impacts gene expression in a cell typedependent manner. Science 325, 1246-1250, doi:10.1126/science.1174148 (2009).

70 Bick, A. G. et al. Inherited Causes of Clonal Hematopoiesis of Indeterminate Potential in TOPMed Whole Genomes. bioRxiv (preprint), doi:10.1101/782748 (2019).

71 Bassett, A. R. et al. Considerations when investigating IncRNA function in vivo. eLife 3, e03058 (2014).

72 Zhou, J. \& Troyanskaya, O. G. Predicting effects of noncoding variants with deep learning-based sequence model. Nat Methods 12, 931-934, doi:10.1038/nmeth.3547 (2015).

73 Buenrostro, J. D., Wu, B., Chang, H. Y. \& Greenleaf, W. J. ATAC-seq: A Method for Assaying Chromatin Accessibility Genome-Wide. Curr Protoc Mol Biol 109, 2129 21-21 29 29, doi:10.1002/0471142727.mb2129s109 (2015).

74 Zhu, J. et al. Genome-wide chromatin state transitions associated with developmental and environmental cues. Cell 152, 642-654, doi:10.1016/j.cell.2012.12.033 (2013).

75 Consortium, E. P. An integrated encyclopedia of DNA elements in the human genome. Nature 489, 57-74, doi:10.1038/nature11247 (2012).

76 Li, H. \& Durbin, R. Fast and accurate short read alignment with Burrows-Wheeler transform. Bioinformatics (Oxford, England) 25, 1754-1760, doi:10.1093/bioinformatics/btp324 (2009).

77 Li, H. et al. The Sequence Alignment/Map format and SAMtools. Bioinformatics 25, 2078-2079, doi:10.1093/bioinformatics/btp352 (2009).

78 Amemiya, H. M., Kundaje, A. \& Boyle, A. P. The ENCODE Blacklist: Identification of Problematic Regions of the Genome. Sci Rep 9, 9354, doi:10.1038/s41598-019-45839-z (2019).

79 Langmead, B. \& Salzberg, S. L. Fast gapped-read alignment with Bowtie 2. Nature methods $\mathbf{9}$, 357-359, doi:10.1038/nmeth.1923 (2012).

80 Liu, J. Z. et al. Association analyses identify 38 susceptibility loci for inflammatory bowel disease and highlight shared genetic risk across populations. Nature genetics 47, 979-986, doi:10.1038/ng.3359 (2015).

81 Wang, G., Sarkar, A., Carbonetto, P. \& Stephens, M. A simple new approach to variable selection in regression, with application to genetic fine mapping. Journal of the Royal Statistical Society: Series B (Statistical Methodology), doi:10.1111/rssb.12388 (2020). biobank-scale datasets. Nat Genet 50, 906-908, doi:10.1038/s41588-018-0144-6 (2018).

83 Zhou, W. et al. Efficiently controlling for case-control imbalance and sample relatedness in largescale genetic association studies. Nat Genet 50, 1335-1341, doi:10.1038/s41588-018-0184-y (2018).

84 Benner, C. et al. Prospects of Fine-Mapping Trait-Associated Genomic Regions by Using Summary Statistics from Genome-wide Association Studies. Am J Hum Genet 101, 539-551, doi:10.1016/j.ajhg.2017.08.012 (2017). 
85 McLaren, W. et al. The Ensembl Variant Effect Predictor. Genome Biol 17, 122, doi:10.1186/s13059-016-0974-4 (2016).

86 Fujita, P. A. et al. The UCSC Genome Browser database: update 2011. Nucleic acids research 39, D876-882, doi:10.1093/nar/gkq963 (2011).

87 Finucane, H. K. et al. Partitioning heritability by functional annotation using genome-wide association summary statistics. Nature genetics 47, 1228-1235, doi:10.1038/ng.3404 (2015).

88 Javierre, B. M. et al. Lineage-Specific Genome Architecture Links Enhancers and Non-coding Disease Variants to Target Gene Promoters. Cell 167, 1369-1384.e1319, doi:10.1016/j.cell.2016.09.037 (2016).

89 Rao, S. S. et al. A 3D map of the human genome at kilobase resolution reveals principles of chromatin looping. Cell 159, 1665-1680, doi:10.1016/j.cell.2014.11.021 (2014).

90 Novakovic, B. et al. $\beta$-Glucan Reverses the Epigenetic State of LPS-Induced Immunological Tolerance. Cell 167, 1354-1368.e1314, doi:10.1016/j.cell.2016.09.034 (2016).

91 Donnard, E. et al. Comparative Analysis of Immune Cells Reveals a Conserved Regulatory Lexicon. Cell Syst 6, 381-394 e387, doi:10.1016/j.cels.2018.01.002 (2018).

92 Burdett, T. et al. The NHGRI-EBI Catalog of published genome-wide association studies., $<$ http://www.ebi.ac.uk/gwas $>$. 